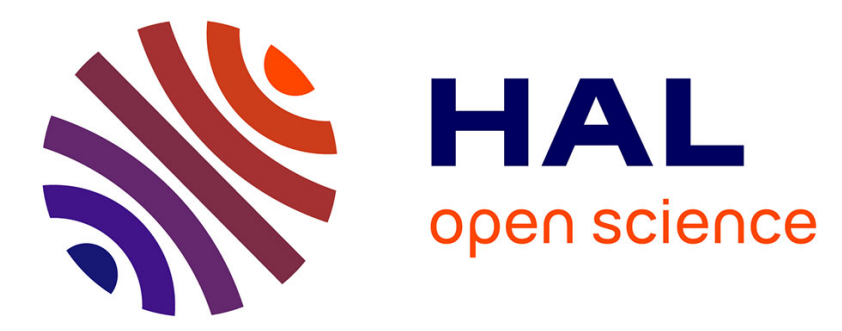

\title{
Perspectives de forte puissance à l'état solide en ondes millimétriques
}

\author{
D. Lippens, M. R. Friscourt, P.A. Rolland, Y. Crosnier
}

\section{To cite this version:}

D. Lippens, M. R. Friscourt, P.A. Rolland, Y. Crosnier. Perspectives de forte puissance à l'état solide en ondes millimétriques. Revue de Physique Appliquée, 1987, 22 (11), pp.1433-1450. 10.1051/rphysap:0198700220110143300 . jpa-00245693

\section{HAL Id: jpa-00245693 https://hal.science/jpa-00245693}

Submitted on 1 Jan 1987

HAL is a multi-disciplinary open access archive for the deposit and dissemination of scientific research documents, whether they are published or not. The documents may come from teaching and research institutions in France or abroad, or from public or private research centers.
L'archive ouverte pluridisciplinaire HAL, est destinée au dépôt et à la diffusion de documents scientifiques de niveau recherche, publiés ou non, émanant des établissements d'enseignement et de recherche français ou étrangers, des laboratoires publics ou privés. 


\title{
Perspectives de forte puissance à l'état solide en ondes millimétriques
}

\author{
D. Lippens, M. R. Friscourt, P. A. Rolland et Y. Crosnier \\ Centre Hyperfréquences et Semiconducteurs, U.A. C.N.R.S. n 287, Bât. P4, U.F.R. d'I.E.E.A., Université \\ des Sciences et Techniques de Lille Flandres Artois, 59655 Villeneuve d'Ascq Cedex, France
}

(Reçu le 16 janvier 1987, révisé le 27 juin 1987, accepté le 30 juin 1987)

\begin{abstract}
Résumé. - Le formidable effort fait actuellement dans les laboratoires sur les composants de type tripôle pose la question du choix d'un composant pour la génération de puissance en ondes millimétriques, actuellement dominée par les dipôles à résistance négative. Dans cet article, nous essayons de répondre à cette question en considérant les composants à effet de champ (MESFET et TEGFET) bipolaire à hétérojonction (HBT), à transfert électronique (diode GUNN) et à ionisation par choc (diodes IMPATT). Nous montrons que dans le bas de la bande millimétrique les performances des composants tripôles en amplificateur de puissance tendent de plus en plus à être comparables à celles obtenues pour les composants dipôles en régime d'oscillation (typiquement $1 \mathrm{~W}$ à $30 \mathrm{GHz}$ ). Par contre pour des fréquences de l'ordre et supérieures à $100 \mathrm{GHz}$ les composants dipôles se révèlent les plus puissants.
\end{abstract}

\begin{abstract}
The tremendous effort devoted to three terminal devices actually in laboratories brings the supremacy of dipoles in question for power generation in the millimeter-wave frequency range. In this paper, we try to answer this question by concentrating on field effect transistors (MESFET and TEGFET), heterojunction bipolar transistor (HBT) transfert electron devices (TED) and impact ionization devices (IMPATT). It is shown that the performances of three terminal devices used in power amplification tend to be comparable with those obtained by means of two-terminal devices (typically $1 \mathrm{~W}$ at $30 \mathrm{GHz}$ ) in the low frequency range. On the other hand, for high frequency operation at and above $100 \mathrm{GHz}$, two terminal devices remain the most powerful avalaible solid state devices.
\end{abstract}

\section{Introduction.}

Au cours de ces dix dernières années, les systèmes millimétriques ont pris une importance grandissante. Les raisons de ce développement sont essentiellement la résolution élevée obtenue pour les applications militaires et la capacité accrue pour les systèmes de télécommunications. Actuellement, la plupart des activités sont centrées sur les fenêtres atmosphériques $35 \mathrm{GHz}$ et $94 \mathrm{GHz}$, le développement aux fréquences de $140 \mathrm{GHz}$ et $220 \mathrm{GHz}$ constituant un objectif à long terme.

Ce développement des applications millimétriques est en grande partie tributaire des performances des composants de puissance. Parmi les solutions avancées, les composants dipôles essentiellement diodes GUNN et diodes IMPATT se sont imposés. Or, l'essor considérable des études relatives aux composants tripôles principalement transistors à effet de champ et bipolaire tend à modifier cette tendance.
Dans cet article, nous nous proposons de faire le point sur cette évolution. Nous nous attacherons tout d'abord à préciser les phénomènes physiques mis en jeu en essayant de mettre en évidence les limitations fondamentales inhérentes au mécanisme de transport de porteurs, d'ionisation par choc et de transfert électronique. Les problèmes technologiques tels que ceux qui influent directement sur les éléments parasites constitueront le deuxième aspect de cette partie. Nous nous efforcerons ensuite de dresser un constat des niveaux de puissance disponibles à l'heure actuelle et ceux que l'on peut prédire raisonnablement pour les quelques années à venir. Nous essayerons en particulier d'apporter des éléments de réponse quant au choix d'un composant de moyenne et de forte puissance. Enfin, nous nous attacherons à présenter les structures nouvelles développées actuellement dans les laboratoires dans le but de pallier aux limitations physiques et technologiques des composants conventionnels. Nous ver- 
rons en particulier les améliorations que l'on peut attendre par l'utilisation de nouveaux matériaux et de structures distribuées.

\section{Limitations physiques et technologiques.}

2.1 Composants Dipoles. - Deux types de composants dipoles sont actuellement utilisés comme source de moyenne et de forte puissance à l'état solide. Ce sont les diodes GUNN et les diodes IMPATT. Nous allons les considérer respectivement.

2.1.1 Diodes IMPATT. - Les composants IMPATT (Impact Ionization Avalanche Transit Time) font intervenir dans leur fonctionnement un processus d'émission de paires électron-trou par avalanche suivi d'un mécanisme de transit des porteurs dans la zone active, constituée par la zone désertée d'une jonction p-n ou métal-semiconducteur. Ces deux effets conjugués font que la partie réelle de l'impédance de la diode est négative pour des fréquences supérieures à une fréquence critique dite d'avalanche [1]. Pour des fonctionnements en gamme d'ondes millimétriques, on observe une diminution de la valeur absolue de la résistance négative dont les causes essentielles sont les suivantes.

Il apparaît tout d'abord que le mécanisme d'avalanche permettant la création de paquet de porteurs n'est pas instantané. En première approximation, le temps de réponse de l'avalanche est d'autant plus faible que la zone de champs électriques élevés, où se produit la multiplication des électrons et des trous, a une longueur réduite [2]. Typiquement pour une zone de multiplication de $0,2 \mu \mathrm{m}$, le temps de réponse de l'avalanche est de l'ordre de la picoseconde. Il en résulte qu'il est de plus en plus difficile de créer des porteurs par avalanche au fur et à mesure que la fréquence de fonctionnement augmente. En pratique, on observe une diminution de l'amplitude des variations temporelles du courant de porteurs créés par ionisation par choc. Cette limitation fondamentale du mécanisme d'avalanche est illustrée sur la figure 1 où nous avons tracé les évolutions temporelles du courant d'avalanche pour des fréquences de fonctionnement de $10 \mathrm{GHz}$ et de $80 \mathrm{GHz}$. Il est clair, au vu de ces résultats, que la non linéarité du phénomène d'avalanche décroit avec la fréquence.

On serait tenté face à cette limitation de diminuer les dimensions de la zone de multiplication. On se heurte cependant à une limitation tout aussi fondamentale, l'« espace noir» [3]. Rappelons en effet qu'un porteur ne peut ioniser que s'il acquiert l'énergie de seuil d'ionisation. L'espace noir est la distance nécessaire à cette acquisition. Deux conséquences importantes vont en résulter. D'une part, on observe au sein de la zone où se produit

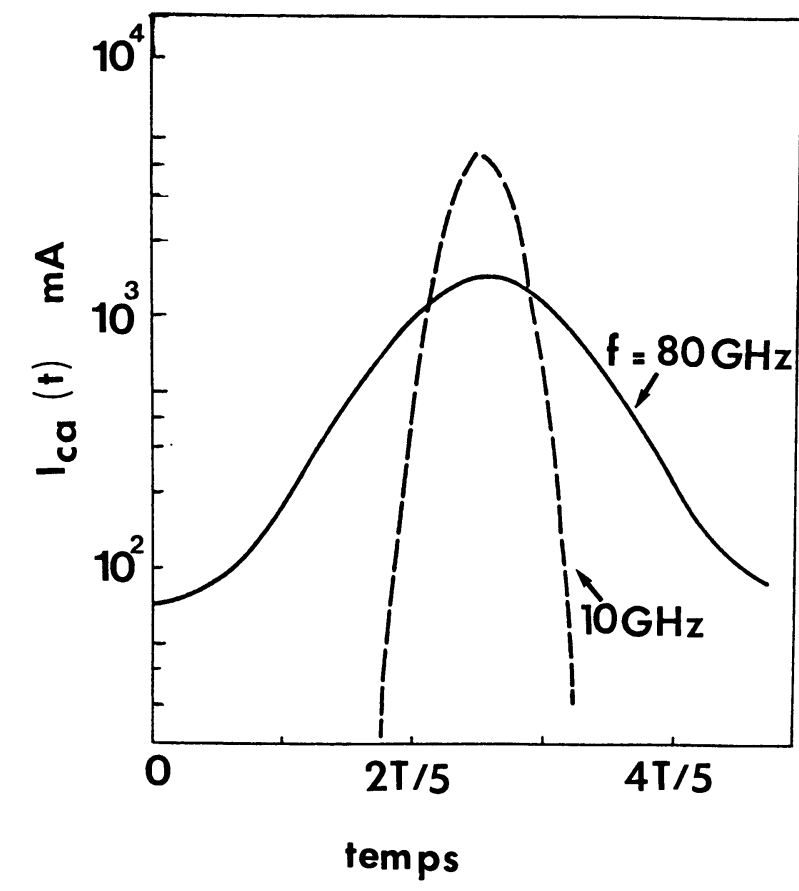

Fig. 1. - Variations temporelles du courant injecté dans la zone de transit pour une diode IMPATT à l'arséniure de gallium.

[Time variations of the avalanche-zone current of GaAs IMPATT diode.]

l'avalanche, l'existence de zones inactives qui limitent une trop grande diminution de zones de multiplication. En pratique, la limite inférieure des dimensions des zones d'avalanche est de l'ordre de $0,1 \mu \mathrm{m}$. D'autre part, les coefficients d'ionisation tracés pour le $\mathrm{Si}$, l'AsGa et l'InP sur la figure 2 vont saturer aux valeurs de champs électriques élevés. Il en résulte une diminution de la non linéarité du phénomène d'avalanche qui est directement liée à la dérivée du coefficient d'ionisation par rapport au champ électrique.

Par ailleurs, la phase du courant des porteurs injectés dans la zone de transit qui vaut $90^{\circ}$ à basses fréquences [4], va se décaler en hautes fréquences en raison de deux phénomènes antagonistes. Il est important de noter tout d'abord que les valeurs de champs électriques pour lesquels on observe un claquage par avalanche deviennent de plus en plus élevées à fréquences croissantes suite aux réductions des dimensions des zones actives. Il en résulte la possibilité de création de porteurs par effet tunnel. Ceci entraîne une diminution de la phase d'injection néfaste à l'obtention de valeurs de résistance négative élevée [5]. En contrepartie, puisque le phénomène de multiplication par avalanche dépend fondamentalement de l'énergie des porteurs, il est relativement intuitif que la création des paires électronstrous va être d'autant plus retardée que la fréquence augmente en raison des effets de relaxation de l'énergie $[6,7]$. 


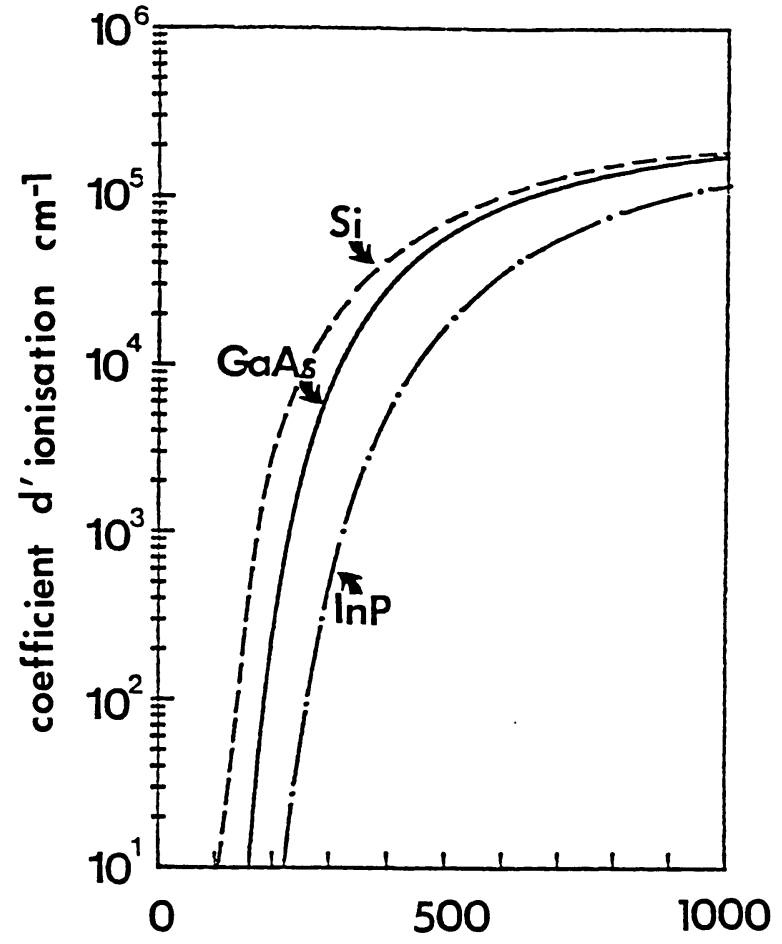

champ électrique $\mathrm{kVmm}^{-1}$

Fig. 2. - Variation des coefficients d'ionisation des électrons en fonction du champ électrique pour les matériaux les plus utilisés en millimétrique.

[Electron ionization rates versus electric field for different materials.]

Si l'on considère à présent le processus de transit, le paramètre qui nous paraît essentiel est la vitesse à laquelle les porteurs se déplacent avant d'être collectés en fin de période hyperfréquence. La figure 3 montre les évolutions des caractéristiques vitessechamp électrique pour les trois matériaux utilisés ou susceptibles d'être utilisés dans la fabrication des diodes IMPATT millimétriques. On constate que la

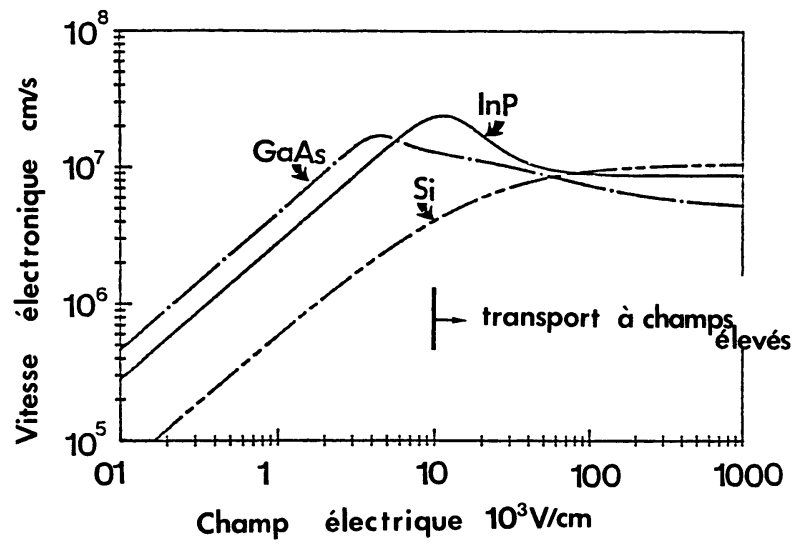

Fig. 3. - Caractéristiques vitesse-champ électrique.

[Electron drift velocity versus electric field.] vitesse de saturation de l'arseniure de gallium est inférieure à celle du phosphure d'indium et du silicium [8]. Il va en résulter que la diminution des dimensions des zones de transit imposée par la condition de temps de transit est plus importante pour l'AsGa que pour les deux autres matériaux. Dans ce cas on observe une diminution du niveau de résistance négative avec la fréquence plus marquée. Ceci s'explique en première approximation par une augmentation de la réactance inversement proportionnelle à la longueur de la zone active.

D'un point de vue technologique, la diminution du niveau de résistance négative mis en évidence précédemment a pour conséquence que le composant devient plus sensible aux résistances parasites de substrat, de contact et de zone active non désertée. La contribution de la résistance de substrat est dans bien des cas déterminante. Dans ce cas, les technologues ont recours à des techniques d'amincissement de substrat dont la valeur nominale devient de l'ordre de $10 \mu \mathrm{m}$, voire inférieure $[9,11]$. En ce qui concerne les conditions de fonctionnement, les densités de courants de polarisation $J_{0}$ sont d'autant plus élevées que la fréquence augmente. Ceci s'explique par la nécessité d'augmenter la fréquence d'avalanche proportionnelle à $J_{0}$. Ainsi pour une diode IMPATT silicium fonctionnant à des fréquences voisines de $220 \mathrm{GHz}$, la densité de courant de polarisation est typiquement de $100 \mathrm{kA} / \mathrm{cm}^{2}$. Un fonctionnement à de telles densités de courant compliquent notablement les problèmes de dissipation thermique. Ceci est d'autant plus vrai pour l'InP qui nécessite également des tensions de polarisation plus élevées en raison des valeurs relativement faibles des coefficients d'ionisation (voir Fig. 2). En pratique, le recours au montage des composants sur radiateur diamant à faible résistance thermique constitue une solution largement employée dans la résolution de ces problèmes [10].

Compte tenu de ces limitations tant physiques que technologiques, nous avons tracé sur la figure 4 l'évolution théorique des rendements et des niveaux de puissance de sortie potentiels pour des diodes IMPATT à double zone de transit en fonction de la fréquence. Ces résultats ont été obtenus par simulation numérique des composants en régime grand signal [12] dont un exemple est donné sur la figure 5. Le niveau d'impédance minimum adaptable est supposé égal à $1 \Omega$. On constate, en accord avec l'analyse précédente, que les performances de l'AsGa sont en deçà de celles calculées pour le silicium et le phosphure d'indium. Dans la bande de fréquence étudiée, les diodes silicium présentent également des performances supérieures à celles de diodes InP.

2.1.2 Diodes GUNN. - La diode GUNN est un composant basé sur l'effet de transfert électronique. 


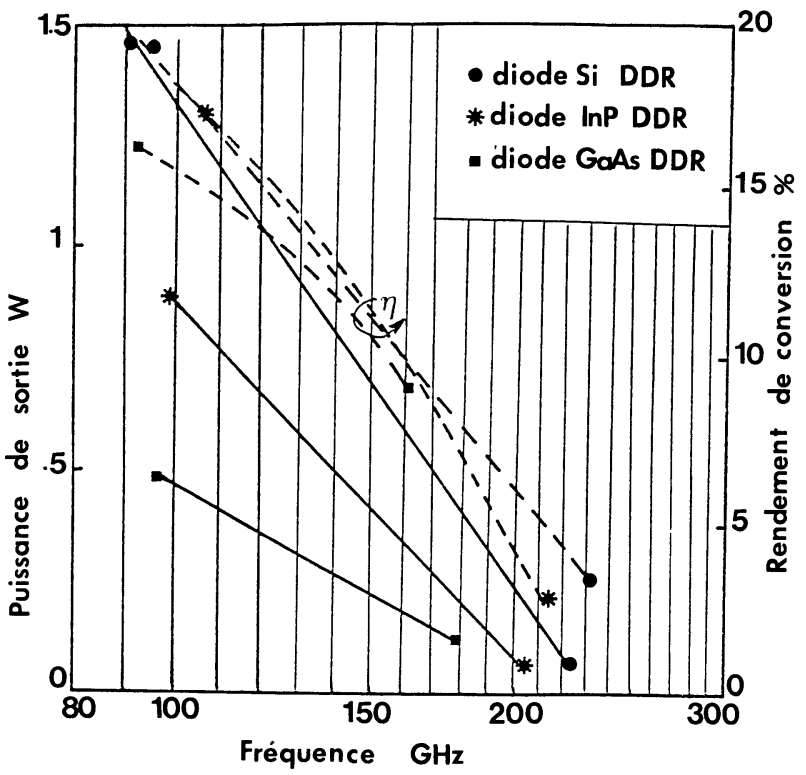

Fig. 4. - Evolution des rendements de conversion et des puissances de sortie théoriques en fonction de la fréquence, obtenue par simulation de diodes $\mathrm{Si}$, InP et GaAs.

[Theoretical conversion efficiency and output power versus frequency (simulation of $\mathrm{Si}, \mathrm{InP}, \mathrm{GaAs}$ IMPATT diodes).]

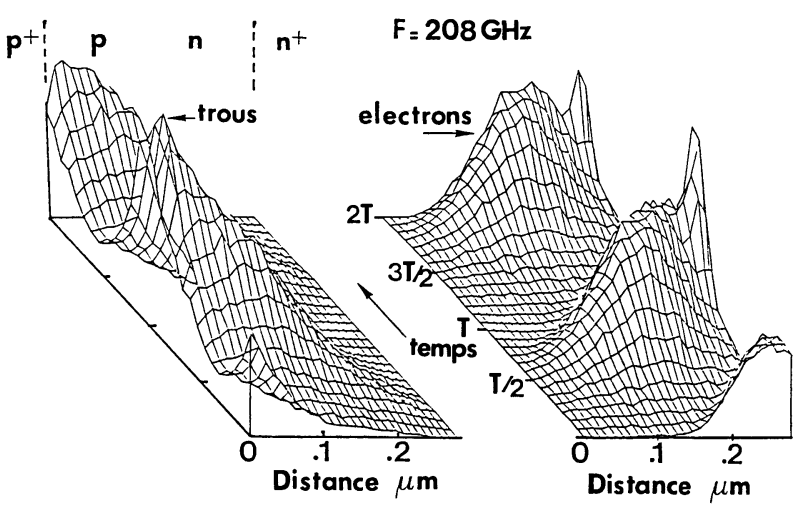

Fig. 5. - Répartitions des densités d'électrons et de trous au cours du temps (simulation microscopique du fonctionnement d'une diode double drift GaAs à $200 \mathrm{GHz}$ ).

[Electron and hole density distribution at different times of the RF cycle (microscopic simulation of a double drift GaAs diode for a $200 \mathrm{GHz}$ operation.]

Le transfert des électrons se produit entre la vallée centrale de la bande de conduction (états de basse énergie à haute mobilité) et les vallées satellites $L$ et $\mathrm{X}$ de la structure de bande (états de haute énergie à faible mobilité). Pour des champs électriques appliqués faibles, les électrons occupent la vallée centrale. Leur vitesse, qui peut atteindre une valeur maximale $v_{\text {pic }}$, est importante du fait de la mobilité bas champ élevée. Dès que le champ tend vers la valeur critique $E_{\text {th }}$ déterminée par l'énergie de séparation entre vallées centrale et latérale $\varepsilon_{\mathrm{S}}$, les électrons transfè- rent vers les états de faible mobilité. Il en résulte une décroissance relativement abrupte de la vitesse moyenne qui devient alors égale à la vitesse de dérive $v_{\text {vallée }}$ dans les vallées satellites. Cette région de mobilité différentielle négative donne lieu à une résistance négative en volume. On obtient ainsi la possibilité de convertir de l'énergie continue en énergie hyperfréquence, le rendement de conversion étant d'autant plus important que le rapport $v_{\text {pic }} / v_{\text {vallée }}$ est élevé [13].

Le transfert électronique n'est cependant pas instantané. L'analyse théorique du transfert des électrons [19] montre en effet que la vitesse de transfert est essentiellement limitée par le temps nécessaire aux électrons pour gagner ou perdre de l'énergie dans la vallée centrale [14]. L'un des moyens de chiffrer ce retard et de voir dans quelle mesure il dépend des propriétés des matériaux, consiste à utiliser l'approximation des temps de relaxation [15]. En première analyse, la valeur moyenne du temps de relaxation de l'énergie peut s'exprimer sous la forme approchée

$$
\tau_{\varepsilon}=k\left(\varepsilon_{\mathrm{S}}-\varepsilon_{0}\right) / q E_{\mathrm{th}} v_{\mathrm{p}}
$$

où : $\varepsilon_{\mathrm{S}}, E_{\mathrm{th}}, v_{\mathrm{p}}$ désignent l'énergie entre vallées centrale et latérale, le champ de seuil et la vitesse pic définis précédemment, $\varepsilon_{0}$ l'énergie thermique, $K$ un coefficient de pondération. Typiquement, les temps de relaxation de l'énergie sont de l'ordre de la picoseconde. Il en résulte qu'en recherchant un fonctionnement aux fréquences millimétriques, le transfert n'ait plus le temps de s'établir. On peut s'attendre dans ce cas à une diminution significative, voire à la disparition complète de la conductivité différentielle négative. En outre, la fréquence caractéristique pour laquelle cette limitation fondamentale intervient dépend du matériau utilisé. Dans ce contexte, il apparaît intéressant de comparer tout d'abord les différents matériaux susceptibles d'être utilisés dans la réalisation des diodes GUNN.

Dans ce but et en première analyse, nous supposons que les échantillons de semiconducteur sont soumis à des champs alternatifs de fréquences élevées et spatialement uniformes. La figure 6 montre comment le déphasage vitesse-champ électrique appliqué évolue en fonction de la fréquence. Ces résultats ont été obtenus par simulation du transport des électrons en y incluant les effets d'inertie décrits précédemment [15]. On peut noter au vu de ces résultats obtenus pour l'AsGa que le déphasage de la modulation de vitesse induit par les variations de champ électrique croît avec la fréquence. A basses fréquences, les variations temporelles de vitesse et de champ électrique sont en opposition de phase et la mobilité différentielle est négative. Aux fréquences élevées la mobilité différentielle décroît et s'annule à la fréquence de coupure définie pour un déphasage égal à $3 \pi / 2$. La fréquence caractéristi- 


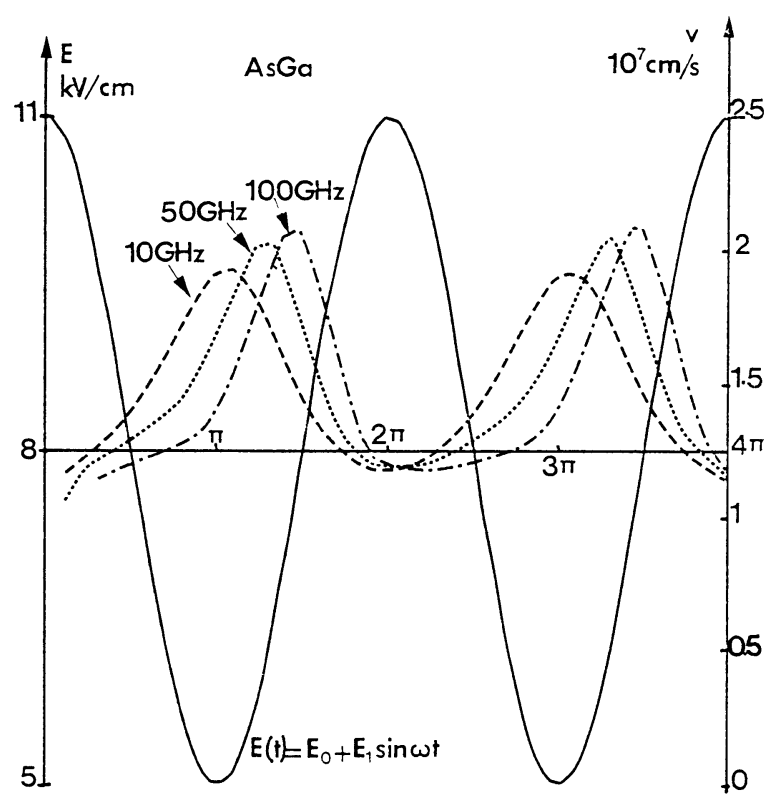

Fig. 6. - Evolution de la mobilité différentielle négative en fonction de la fréquence (transfert électronique dans l'AsGa).

[Evolution of the negative differential mobility as a function of frequency (GaAs transferred electron effect).]

que pour laquelle la mobilité différentielle négative disparaît est d'autant plus élevée que le temps de relaxation de l'énergie est faible. La figure 7 montre les évolutions des temps de relaxation de l'énergie en fonction de l'énergie moyenne du gaz électronique. On peut noter que l'InP présente des valeurs de temps de relaxation plus faibles comparativement à celles obtenues pour le GaAs et pour le GaInAs. On peut donc en conclure que l'InP est plus apte à monter en fréquence. Ce résultat est confirmé par les résultats donnés sur la figure 8 qui montre les variations théoriques du rendement de conversion

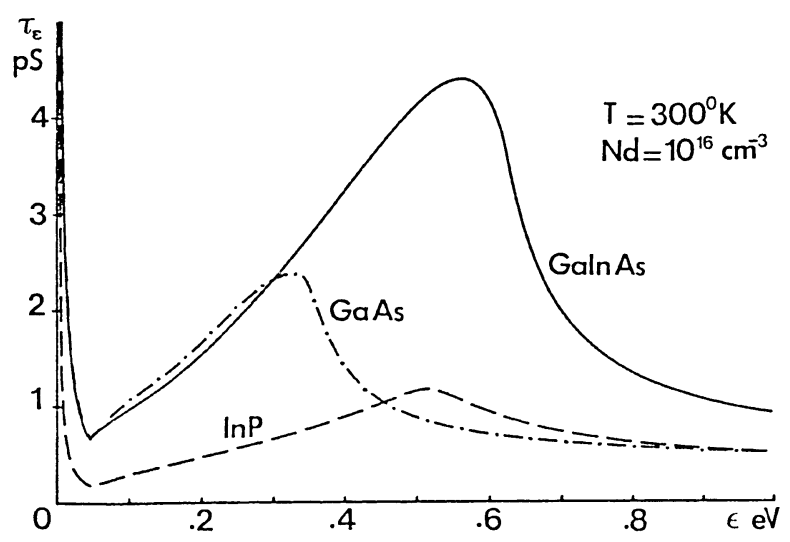

Fig. 7. - Variations des temps de relaxation de l'énergie en fonction de l'énergie moyenne des électrons.

[Energy relation time as a function of electron average energy.]

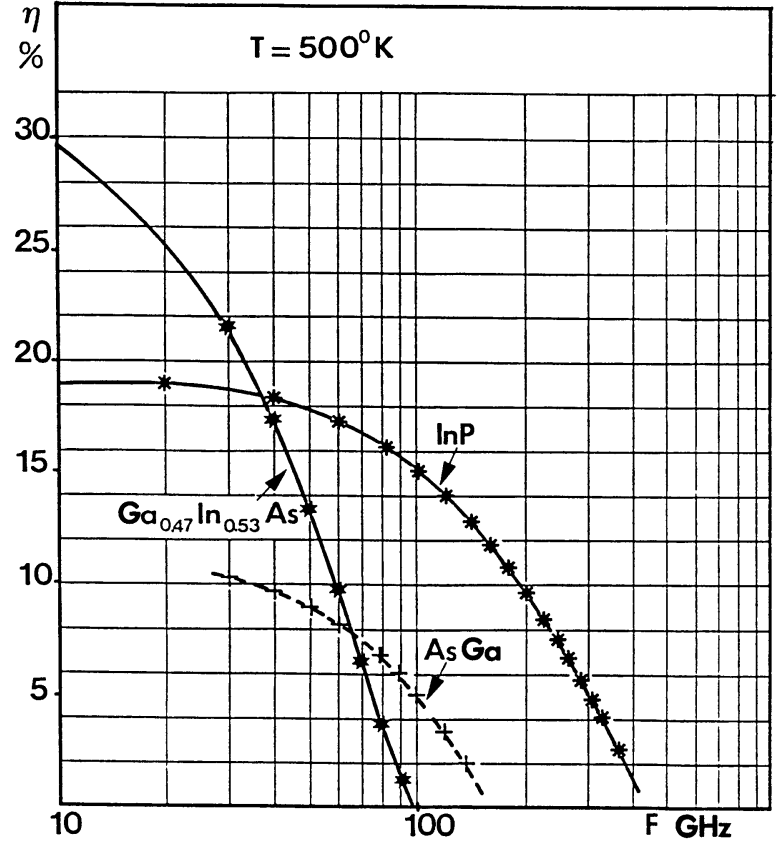

Fig. 8. - Variations théoriques des rendements de conversion en fonction de la fréquence pour des conditions de champ électrique uniforme spatialement.

[Calculated frequency dependence of conversion efficiency (uniform field condition).]

en fonction de la fréquence pour ces trois matériaux, obtenues pour des conditions de champs électriques spatialement uniformes [15].

$\mathrm{Si}$ les études de l'effet de transfert en volume permettent d'appréhender les limitations fondamentales des composants GUNN, les conditions de champs uniformes supposées précédemment ne sont pas réalisées en pratique pour des composants de structure conventionnelle de type $\mathrm{n}^{+} \mathrm{nn}^{+}$. Le fonctionnement de ces composants est illustré sur la figure 9 [16]. L'effet de mobilité différentielle négative entraîne la formation d'une couche d'accumulation qui transite de la cathode à l'anode. Les diodes GUNN sont par conséquent des composants à temps de transit dont la fréquence de fonctionnement est en première approximation inversement proportionnelle à la longueur de la zone active. Ainsi, les dimensions de la zone active d'une diode fonctionnent à $100 \mathrm{GHz}$ sont typiquement de l'ordre du micron. Dans ces conditions, les limitations physiques propres aux composants microniques peuvent alors être mises en évidence [14-17]. Ainsi on peut constater sur la figure 9 que l'énergie de seuil $E_{\text {th }}$ n'est acquise en moyenne que pour une distance à la cathode proche de $50 \%$ de la zone $\mathrm{N}$. A cette abscisse, on observe la formation d'une couche d'accumulation qui résulte de la chute de la vitesse électronique due au transfert intervallée. Cette zone morte introduit une résistance série positive qui diminue de façon significative la résistance négative 

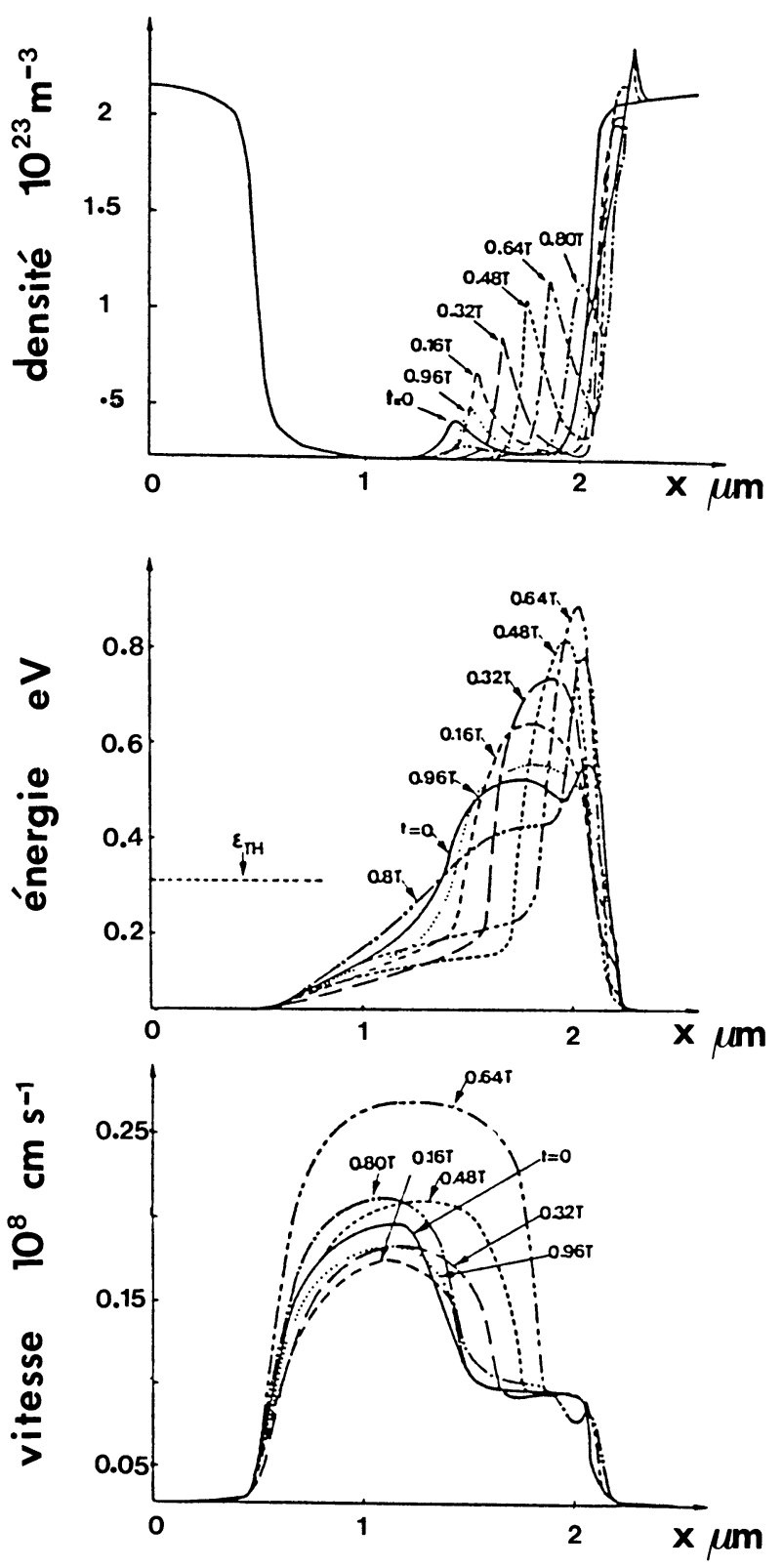

Fig. 9. - Mécanismes de fonctionnement des diodes Gunn $\mathrm{n}^{+} \mathrm{nn}^{+}$.

[Operating mechanisms of $\mathrm{n}^{+} \mathrm{nn}^{+}$Gunn diodes.]

induite par le reste de la zone active. Ce résultat est illustré sur la figure 10 qui montre les variations spatiales de la résistance présentée par le composant à $100 \mathrm{GHz}$. Sur la figure 11 nous présentons l'évolution fréquentielle des rendements de conversion théoriques pour des composants de type $\mathrm{N}^{+} \mathrm{NN}^{+}$ respectivement pour le GaInAs, l'InP et l'arséniure de gallium [18], [20]. Pour ce dernier matériau, les performances calculées en régime d'extraction d'harmonique 2 sont également reportées compte tenu des difficultés d'utilisation de diodes GaAs fonctionnant en mode fondamental [13]. On peut constater que les tendances fréquentielles mises en évidence dans l'analyse simplifiée d'un fonctionne-
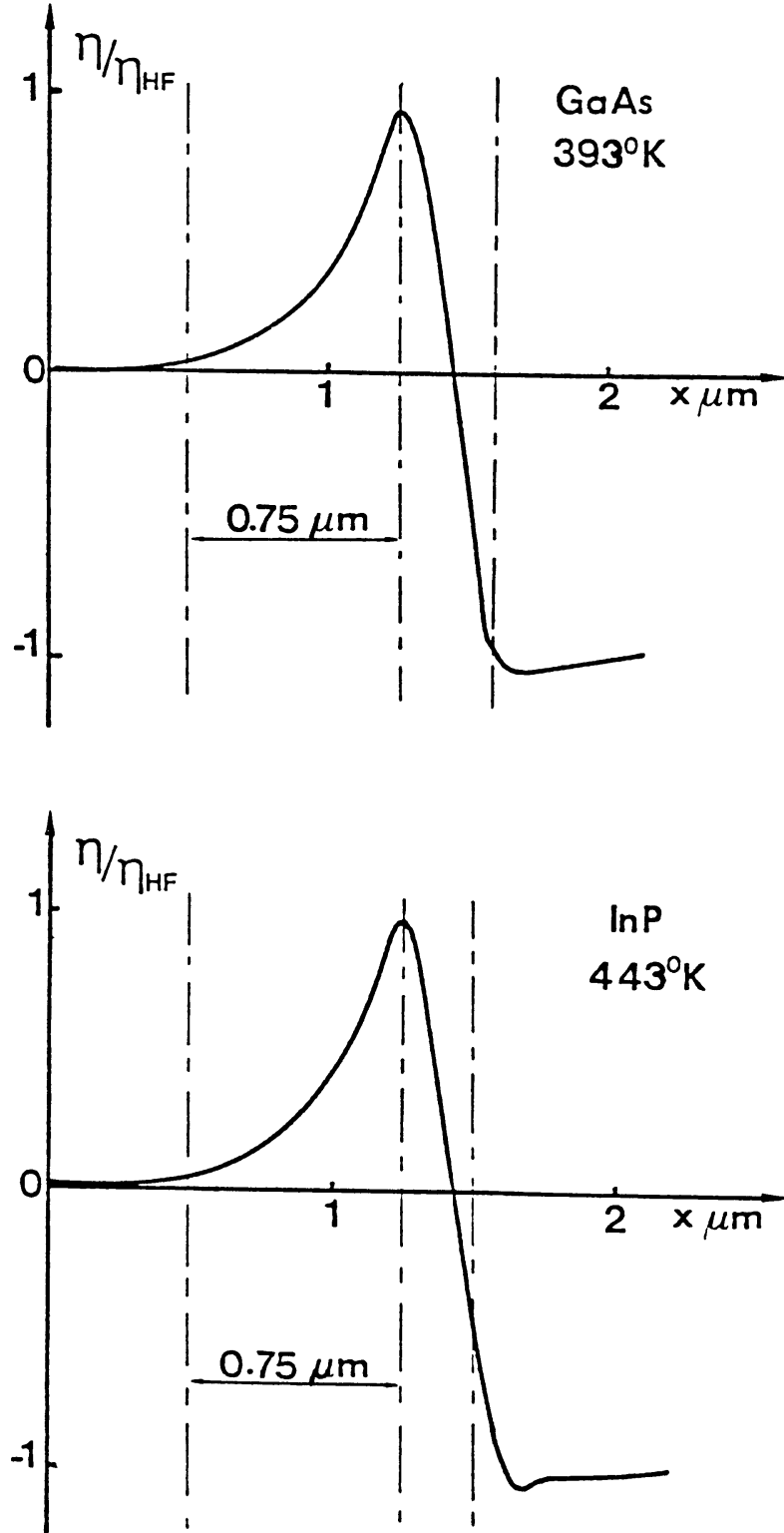

Fig. 10. - Mise en évidence de la zone morte dans les composants Gunn. ( $\eta$ et $\eta_{\mathrm{HF}}$ sont respectivement les rendements de conversion au point $\mathrm{x}$ et émis.)

[Illustration of the dead zone in Gunn devices ( $\eta$ and $\eta_{\mathrm{HF}}$ are the efficiencies at $\mathrm{x}$ point and the conversion efficiency respectively).]

ment en champ uniforme sont ici conservées. Néanmoins, il apparaît que quantitativement les rendements de conversion sont nettement plus faibles dans un fonctionnement à couche d'accumulation. Typiquement, le rendement d'émission est divisé par un facteur deux. On peut donc en conclure qu'un fonctionnement en champ uniforme est plus favorable à l'obtention de rendements élevés. Nous reviendrons sur ce sujet dans le paragraphe 3 .

$\mathrm{Si}$ l'on considère à présent très brièvement les problèmes technologiques, deux difficultés majeures se posent en pratique. En premier lieu, les compo- 


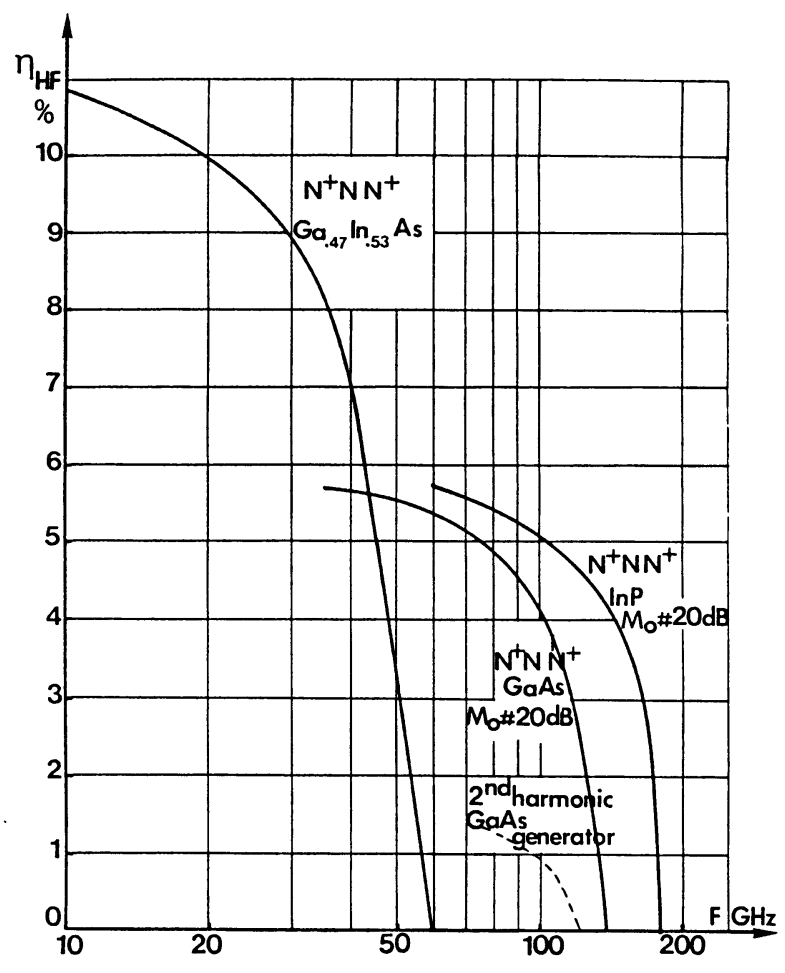

Fig. 11. - Rendements théoriques de conversion pour des composants Gunn $\mathrm{n}^{+} \mathrm{nn}^{+}$en fonction de la fréquence.

[Calculated conversion efficiency of $\mathrm{n}^{+} \mathrm{nn}^{+}$Gunn diodes versus frequency.]

sants présentent une résistance négative faible ce qui signifie que les résistances parasites doivent être comme dans le cas des diodes IMPATT minimisées. En second lieu, puisque les rendements de conversion ne sont que de quelques pourcents pratiquement la totalité de la puissance continue doit être évacuée. De plus, du fait que la plupart des paramètres matériaux évoluent de façon sensible avec la température, il est nécessaire d'effectuer une optimisation de la section de la diode dont dépend la résistance thermique afin de limiter l'élévation de température. Ceci est d'autant plus vrai pour les diodes InP qui nécessitent une puissance de polarisation importante. En pratique, un certain nombre de solutions concernant la géométrie des composants (structure en anneau ou en croix) et l'intégration des dissipateurs thermiques ont été proposées [13].

\subsection{COMPOSANTS TRIPÓLES.}

2.2.1 Transistors à effet de champ MESFET. - Les composants MESFET ont fait l'objet d'un très grand nombre d'études. En conséquence, nous limiterons notre présentation aux deux aspects suivants. D'une part nous essaierons de mettre en évidence les paramètres clés qui limitent la montée en fréquence de ces dispositifs en terme d'évolution fréquentielle du gain en puissance. D'autre part nous nous proposons de soulever les problèmes relatifs à la puissance maximum de sortie délivrée par le composant.

En première analyse, l'aptitude d'un composant de type . FET à monter en fréquence peut être évaluée à partir de la fréquence de coupure (fréquence pour laquelle le gain vaut 1 ) du gain en courant $f_{\mathrm{T}}$ égale à $g_{\mathrm{m}} / 2 C_{\mathrm{gs}}$ où $g_{\mathrm{m}}$ est la transconductance et $C_{\mathrm{gs}}$ la capacité grille source. Moyennant un certain nombre d'approximations, en particulier en négligeant les effets de bord de grille, on montre que $f_{\mathrm{T}}$ est inversement proportionnelle au temps de transit sous la grille. Il en résulte qu'un transistor fonctionne à des fréquences d'autant plus élevées que la vitesse moyenne des porteurs est importante et que la longueur de grille est faible. Les procédés de masquage électronique permettent actuellement de réaliser des grilles très largement submicroniques $(0,25$ à $0,5 \mu \mathrm{m}$ de longueur de grille) .

Il est important de noter toutefois que pour des longueurs de grille aussi faibles, les dimensions et les temps de réponse des composants deviennent comparables aux libres parcours moyens et au temps de libre parcours moyen des porteurs. Il en résulte que le régime stationnaire qui traduit un équilibre entre l'action du champ électrique et les interactions que les porteurs subissent avec le réseau cristallin n'est plus atteint. Les grandeurs physiques vitesse et énergie présentent alors des effets de relaxation vers leurs états stationnaires conduisant en particulier à des effets de survitesse. La détermination du temps de transit effectif sous la grille n'est par conséquent pas directe. Cependant un certain nombre d'informations sur les paramètres matériaux prépondérants dans l'optimisation de la vitesse de transit peuvent être obtenues à partir du critère simple suivant.

La vitesse de transit sera d'autant plus élevée que l'on retarde le transfert des porteurs dans les vallées latérales. En effet, dès que ce transfert est réalisé les porteurs se déplacent à la vitesse limite et les critères relatifs au transit sont alors les mêmes que pour les composants dipôles. A partir de cette référence d'énergie, le temps de transit sous la grille, en régime de mobilité, peut être approximé par la relation [21] :

$$
\tau=q L_{\mathrm{g}}^{2} / \mu_{0} \Delta \varepsilon_{\Gamma \mathrm{L}}
$$

où $\mu_{0}$ désigne la mobilité à bas champ et $\Delta \varepsilon_{\Gamma \mathrm{L}}$ l'énergie intervallées. Sur la figure 12 nous avons comparé les variations de la fréquence de transit $\left(F_{\mathrm{t}}=1 / 2 \pi \tau\right)$ déterminée à partir d'un calcul plus exact prenant en compte en particulier les effets de dynamique non stationnaire [21] pour le $\mathrm{Ga}_{0,47} \mathrm{In}_{0,53} \mathrm{As}$, le $\mathrm{GaAs}$ et l'InP. On constate que le GaInAs est un matériau très intéressant pour monter en fréquence. Il devrait cependant être limité en puissance en raison d'une tension de claquage par avalanche faible.

Si l'on considère à présent l'évolution fréquentielle 


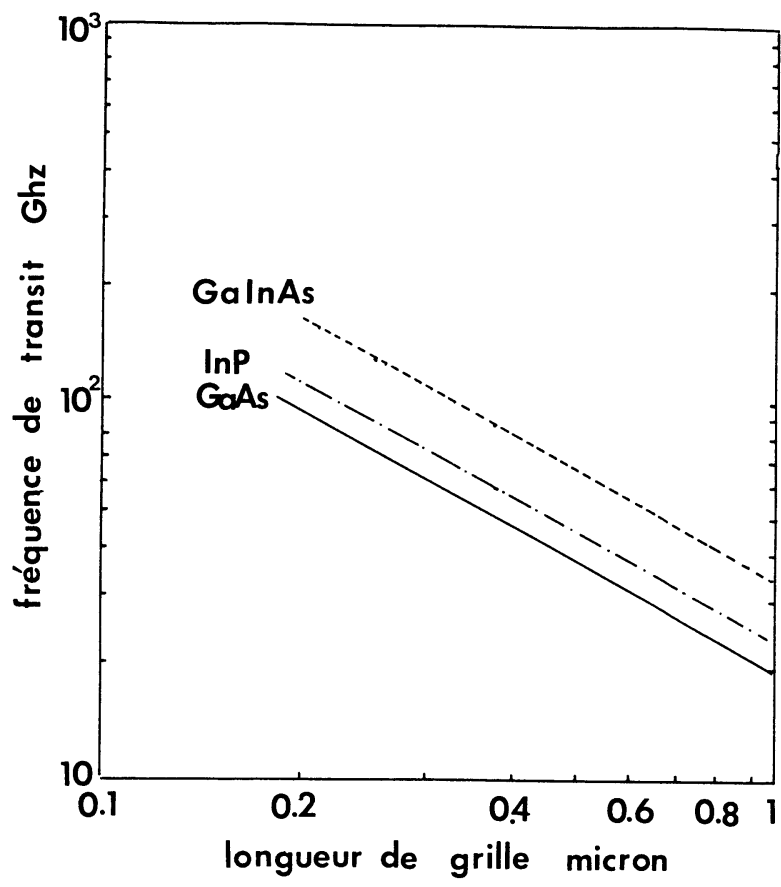

Fig. 12. - Fréquence de transit en fonction de la longueur de grille.

[Transit frequency as a function of gate length.]

du gain en puissance, la fréquence de gain unitaire est donnée par la relation [22]:

$$
\begin{aligned}
F_{\max }=F_{\mathrm{t}} /[ & {\left[g_{\mathrm{d}}\left(R_{\mathrm{i}}+R_{\mathrm{s}}+R_{\mathrm{g}}+\frac{\omega_{\mathrm{T}} L_{\mathrm{s}}}{2}\right)+\right.} \\
& \left.+2 \omega_{\mathrm{T}} C_{\mathrm{gd}}\left(R_{\mathrm{i}}+R_{\mathrm{s}}+2 R_{\mathrm{g}}+\omega_{\mathrm{T}} L_{\mathrm{s}}\right)\right]^{1 / 2}
\end{aligned}
$$

où $g_{\mathrm{d}}$ est la conductance de sortie, $R_{\mathrm{i}}, R_{\mathrm{s}}$ et $R_{\mathrm{g}}$ les résistances de canal non déserté, de source et de grille, $C_{\mathrm{gd}}$ la capacité de contre réaction grille drain, $L_{\mathrm{s}}$ l'inductance de source. Il en résulte que l'augmentation des performances fréquentielles des transistors passe par la diminution des valeurs de ces différentes grandeurs. Dans ce but, les efforts portent actuellement sur l'optimisation des niveaux de dopages, de la géométrie des composants et de la structure du canal, ainsi que le développement de techniques d'auto-alignement et de mise à la masse de la source permettant en particulier de minimiser les éléments parasites $R_{\mathrm{s}}$ et $L_{\mathrm{s}}$. Compte tenu de ces améliorations possibles un bon ordre de grandeur de la fréquence $F_{\max }$ consiste à prendre une valeur égale à $2 F_{\mathrm{t}}$ pour laquelle on peut dire que les éléments de technologie ont été maîtrisés. Sur ce critère il est raisonnable de penser que la valeur de la fréquence $F_{\max }$ puisse être bien supérieure à $100 \mathrm{Ghz}$. Ceci est par ailleurs confirmé par la mise en évidence expérimentale d'oscillations à $110 \mathrm{Ghz}$ [23]. Sur cette base, l'on peut espérer des composants MESFET de puissance dans le bas de la bande des ondes millimétriques.

Si l'on considère à présent les limitations de la puissance de sortie nous pouvons écrire en première approximation que celle-ci est données par la relation :

$$
P_{\mathrm{s}}=\frac{1}{2} \Delta V_{\mathrm{ds}} \Delta I_{\mathrm{ds}} \cos \psi
$$

où $\Delta V_{\mathrm{ds}}$ et $\Delta I_{\mathrm{ds}}$ désignent les excursions de tensions et de courants respectivement et $\psi$ le déphasage entre ces deux grandeurs. On retrouve le critères bien connus des composants de puissance : la puissance délivrée par le composant dépend de façon directe d'une part de la tension $V_{\mathrm{ds}}$ qu'il est possible d'appliquer avant d'observer le claquage par avalanche et d'autre du courant maximum admissible, fonction du niveau de dopage. Pratiquement, la tension $V_{\mathrm{ds}} \max$ fait intervenir deux aspects. En premier lieu, le matériau utilisé joue un rôle prépondérant. il justifie en particulier le choix de matériaux à faible coefficient d'ionisation. En second lieu la géométrie du composant influe sur les caractéristiques de tensions maximales. Ce dernier point est illustré sur la figure 13 où nous montrons les variations de la tension de claquage en fonction de l'angle de creusement de canal [24]. Il est ainsi difficile

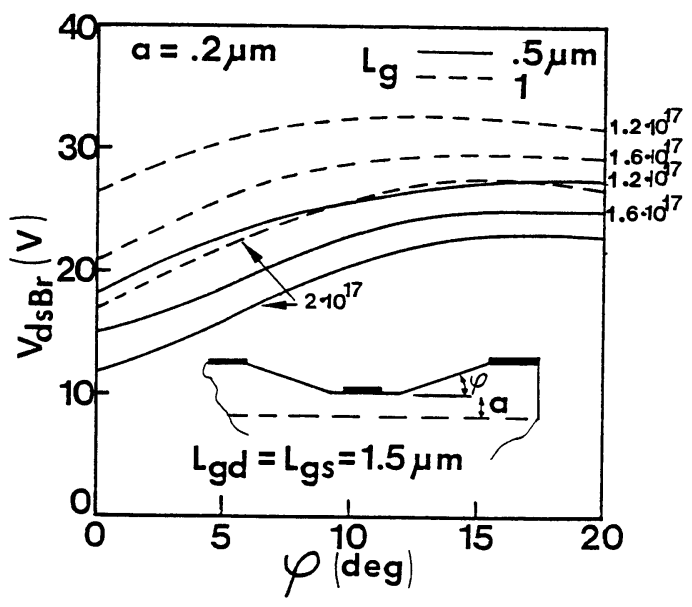

Fig. 13. - Tension de claquage par avalanche en fonction de l'angle de creusement du canal des composants MESFET. Pour différentes longueurs de grilles et différents niveaux de dopage du canal.

[Avalanche breakdown voltage versus recess angle of MESFET devices, for different gate lengths and doping levels.]

d'établir des règles générales dans cette étude des structures pour lesquelles on cherche à éviter des valeurs trop élevées de champ électrique entraînant l'initialisation du phénomène d'ionisation par choc. On peut cependant noter que les objectifs de fonctionnement à courant élevé et tension élevée peuvent être contradictoires dans la mesure où l'augmentation des valeurs de dopage de la zone active conduit à des valeurs très élevées de champ électrique à 
l'interface grille - semiconducteur et donc à une diminution des valeurs de tensions admissibles.

En outre, il est important de noter que l'optimisation de $V_{\mathrm{ds}}$ n'est pas suffisante. En effet, la puissance délivrée par le composant dépend de l'impédance de sortie du transistor traduit par le terme $\cos \psi$ de la relation précédente. La figure 14 montre les variations expérimentales de $\cos \psi$ en fonction de la tension $V_{\mathrm{ds}}$ si l'on considère un fonctionnement en régime linéaire, à $1 \mathrm{~dB}$ de compression, en régime de saturation pour deux transistors fonctionnant en bande $\mathrm{X}$ [25]. L'évolution du terme cos $\psi$ en fonction de la tension $V_{\text {Ds }}$ que l'on observe quel que soit le régime de fonctionnement peut être reliée aux variations de l'impédance de sortie. Les variations de cette impédance en fonction de $V_{\mathrm{DS}}$ sont régies par des effets physiques connus telle que l'extension de la zone désertée vers le drain.
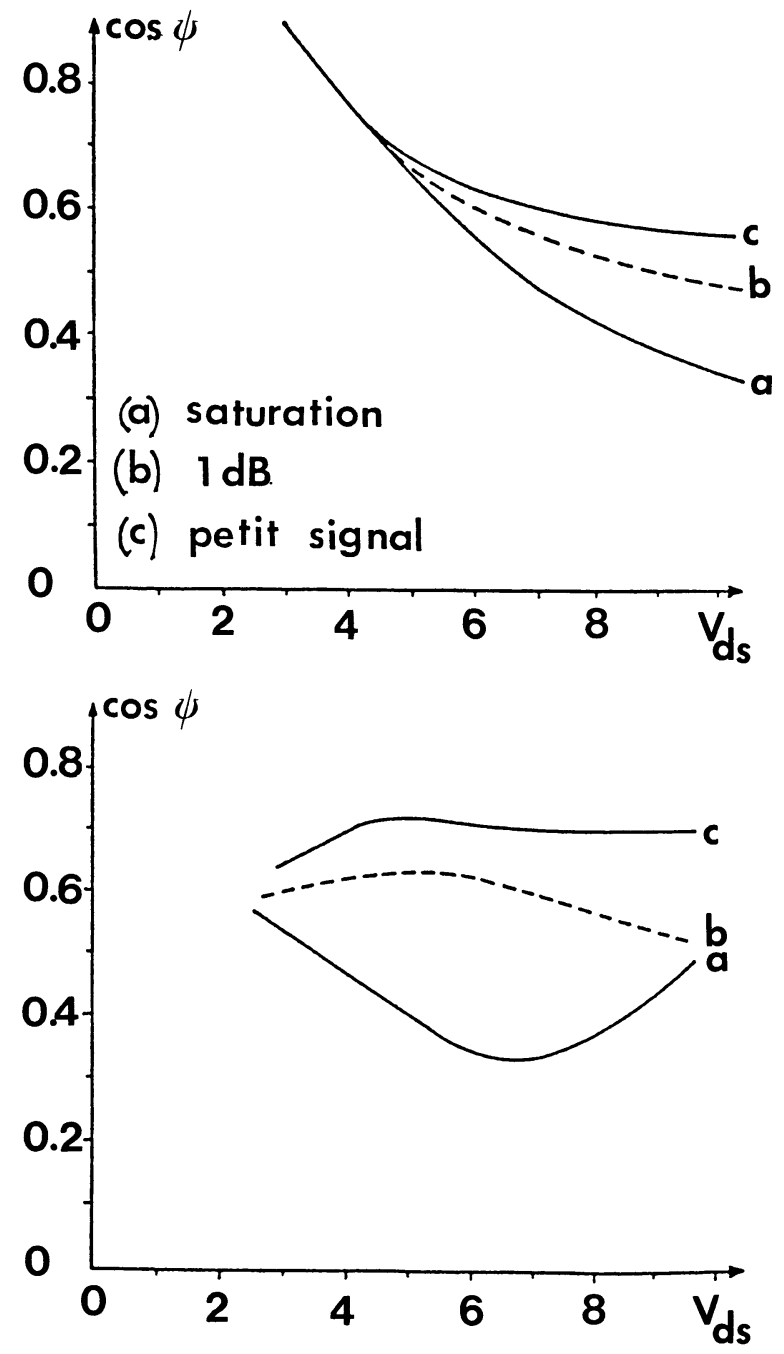

Fig. 14. - Variations expérimentales de $\cos \psi$ (voir texte) en fonction du niveau de puissance à l'entrée et de la tension $V_{\mathrm{ds}}$.

[Experimental variations of $\cos \psi$ (see text) versus input power level and $V_{\mathrm{ds}}$ voltage.]
Bien que les fréquences de fonctionnement soient pour cet exemple relativement faibles, il est clair que l'impédance de sortie joue un rôle prépondérant dans l'optimisation des composants de puissance. Ici encore il peut être recherché des objectifs relativement antagonistes. Ainsi on montre que des transistors présentant une conductance de sortie relativement élevée peuvent présenter un bon comportement en puissance [25]. Ceci est en contradiction avec la recherche d'un fonctionnement à fréquence élevée pour laquelle on vise à minimiser $g_{\mathrm{d}}$.

2.2.2 Transistors TEGFET. - Les transistors à gaz d'électrons bidimensionnel sont également très largement étudiés. Rappelons cependant très brièvement que la réalisation de ces composants fait intervenir les techniques de modulation de dopage visant à introduire des porteurs libres dans un matériau quasi intrinsèque. A l'aide d'une hétérojonction isotype constituée de matériaux présentant des valeurs de bande interdite et de dopage respectivement élevées puis faibles il est possible de créer un gaz d'électrons bidimensionnel. Le transport des porteurs ne fait pas intervenir les collisions avec les impuretés ionisées d'où la possibilité de réaliser des couches à haute mobilité. De cette présentation succincte nous pouvons tirer deux conclusions.

Tout d'abord les performances fréquentielles des transistors TEGFET sont potentiellement plus élevées comparativement aux transistors MESFET en raison des vitesses de transit sous la grille plus importantes. Ce point est illustré sur la figure 15 où nous montrons les variations de la fréquence $F_{\mathrm{t}}$ en fonction de la longueur de grille respectivement pour des transistors MESFET et TEGFET que

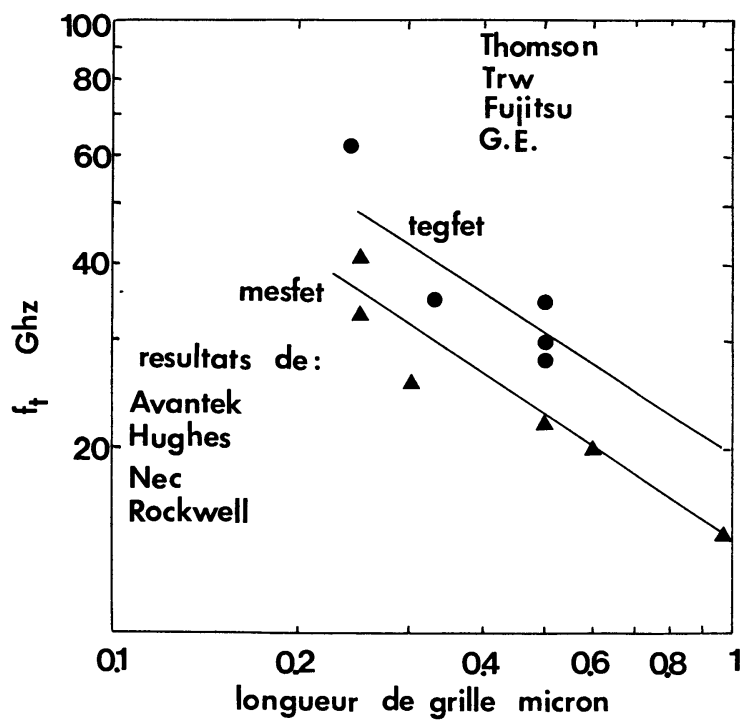

Fig. 15. - Comparaison des fréquences $F_{\mathrm{t}}$ des transistors MESFET et TEGFET.

[Frequency dependence of $F_{\mathrm{t}}$ for MESFET and TEGFET devices.] 
l'on peut mettre en évidence à partir de réalisations expérimentales.

En contrepartie, le confinement des porteurs à l'hétérojonction a pour conséquence de limiter le courant admissible $I_{\mathrm{ds}} \max$ et par conséquent la puissance admissible par le composant. Ainsi, la puissance de saturation d'un transistor à simple hétérojonction fonctionnant à $30 \mathrm{GHz}$ est de $0,2 \mathrm{~W} / \mathrm{mm}$ alors qu'il semble raisonnable d'espérer des puissances de l'ordre de $0,4 \mathrm{~W} / \mathrm{mm}$ pour un dispositif MESFET. Ceci est d'autant plus vrai que la densité de charge surfacique diminue au fur et à mesure que le champ électrique parallèle à l'hétérojonction augmente. On peut le constater sur la figure 16 issue de la référence [26]. On constate qu'en grand signal, condition rencontrée en amplification de puissance, il est relativement difficile d'obtenir des densités superficielles de charge supérieures à $5 \times 10^{11} \mathrm{~cm}^{-2}$.

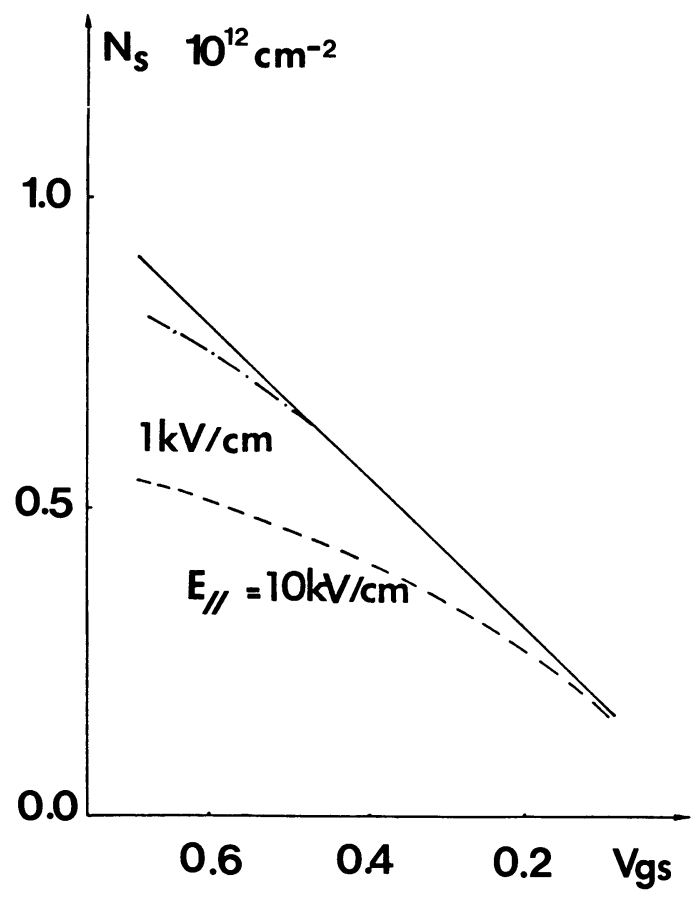

Fig. 16. - Charge superficielle $n_{\mathrm{s}}$ en fonction de $V_{\mathrm{gs}}$ dans le TEGFET GaAs/GaAlAs.

[Interface carrier density versus $V_{\mathrm{gs}}$ voltage in GaAs/GaAlAs TEGFET.]

L'un des moyens d'augmenter les valeurs de courant admissibles consiste à réaliser des composants TEGFET à multiples hétérojonctions [27, 29]. La figure 17 schématisent un exemple de transistor à puits quantiques. A titre d'exemple un transistor de puissance comportant 6 canaux permet d'obtenir un courant maximum admissible entre drain et source de $600 \mathrm{~mA} / \mathrm{mm}$ [28]. La puissance délivrée à l'aide de ce composant peut atteindre $0,6 \mathrm{~W} / \mathrm{mm}$ dans le

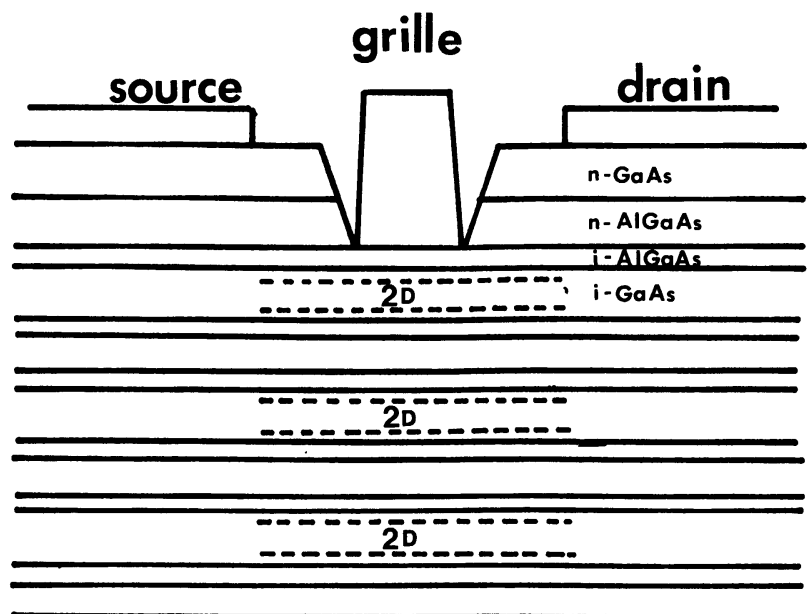

\section{tampon}

\section{substrat}

Fig. 17. - Structure TEGFET à multiples puits quantiques.

[Multiquantum well TEGFET structure.]

bas de la bande millimétrique. Si ces niveaux de puissance sont extrêmement encourageants il est important de noter cependant qu'en pratique les développements de grille dans la direction transversale sont limités pour deux raisons essentielles. Tout d'abord il apparaît qu'il devient de plus en plus difficile d'adapter le composant à fréquences croissantes. Ensuite les effets de propagation suivant la direction transversale de grille vont devenir de plus en plus significatifs aux fréquences très élevées [30]. Il en résulte des déphasages au niveau des signaux de commande de grille en règle générale non constructifs et donc préjudiciales à l'obtention de puissance de sortie élevée. L'utilisation de multiples doigts de grille permet de résoudre en partie ce dernier point au détriment toutefois d'une complication notable dans la réalisation des transistors.

Dans un tout autre esprit, l'utilisation d'autres matériaux constitue une voie de recherche également très prometteuse. A titre d'exemple, citons les résultats obtenus aux fréquences millimétriques pour une structure TEGFET

$$
\mathrm{Ga}_{0,85} \mathrm{In}_{0,15} \mathrm{As} / \mathrm{Ga}_{0,85} \mathrm{Al}_{0,15} \mathrm{As} \text {. }
$$

Une densité de puissance de sortie de $0,43 \mathrm{~W} / \mathrm{mm}$ et un gain associé de $3 \mathrm{~dB}$ ont été obtenus pour des fréquences de l'ordre de $60 \mathrm{GHz}$ [31].

2.2.3 Transistors bipolaires à hétérojonction. - Les études relatives au fonctionnement, aux fréquences millimétriques, des transistors bipolaires à hétéro- 
jonction (HBT) sont encore trop fragmentaires [34] pour que l'on puisse prédire un développement de ces composants comparable à celui des FET et TEGFET. Cependant, du point de vue de la génération de puissance hyperfréquence à des fréquences les plus élevées possibles, les transistors bipolaires à simple et double hétérojonctions [36] présentent un certain nombre d'avantages potentiels comparativement aux autres composants tripôles qui découlent principalement de la structure verticale.

Tout d'abord, il est possible de contrôler les dimensions des zones de commande sans avoir recours, comme c'est le cas pour les transistors à effet de champ, à des techniques de lithographie avancées. Ainsi l'épaisseur de la base peut être réduite à des dimensions inférieures au dixième de micron par épitaxie par jet moléculaire. Ensuite, la composition des matériaux de l'hétérostructure peut être ajustée afin de contrôler séparément les courants d'électrons et de trous. Il en résulte que les électrons peuvent être injectés à vitesse élevée dans la base où ils transitent sans collision avec phonon (transport balistique). La fréquence de transit est par conséquent théoriquement très élevée. De plus par opposition aux structures planar, l'ensemble de la surface d'émetteur supporte le courant sous réserve que la largeur des doigts d'émetteur reste raisonnable (typiquement 1 à $2 \mu \mathrm{m}$ ) de manière à éviter l'apparition de la défocalisation [38]. Il en découle que potentiellement le HBT peut être utilisé en amplification de puissance.

A titre d'exemple, l'aptitude du HBT à fonctionner aux fréquences millimétriques est confirmée par les travaux publiés dans la référence [37] pour lesquels une fréquence $F_{\mathrm{t}}$ de $45 \mathrm{GHz}$ est obtenue. Par ailleurs, il a été démontré la possibilité de générer une puissance normalisée de $2,5 \mathrm{~W} / \mathrm{mm}$ de longueur d'émetteur en bande $X$ [32]. Ces résultats apparaissent particulièrement encourageants si on les compare aux meilleurs résultats MESFET pour lesquels la puissance normalisée se situe autour de $1 \mathrm{~W} / \mathrm{mm}$ en bande $\mathrm{X}$.

L'extension de ces études aux fréquences millimétriques se heurte cependant à l'heure actuelle à des difficultés essentiellement technologiques. En effet, la fréquence $F_{\max }$ est pour les structures réelles du même ordre de grandeur voire inférieure à $F_{\mathrm{t}}$. Dans ce contexte, les études portent actuellement sur la recherche de procédés technologiques permettant de diminer les éléments parasites du transistor. En particulier, de nombreux travaux montrent la possibilité de réduire notablement la résistance extrinsèque de base par des techniques d'auto-alignement de l'émetteur et d'optimisation des contacts ohmiques sur semiconducteur $\mathrm{p}$ fortement dopé $[33,35]$. Si ces études aboutissent, il ne semble pas utopique d'espérer obtenir un $F_{\mathrm{t}}$ de $50 \mathrm{Ghz}$ et un $F_{\max }$ de $140 \mathrm{GHz}$ [34].

\section{Evolution comparative des niveaux de puissance.}

Sur la figure 18 nous présentons les variations fréquentielles des niveaux de puissance émise des composants Gunn InP et $\mathrm{GaAs}$ et des composants IMPATT $\mathrm{Si}$ et $\mathrm{GaAs}$ qu'il est possible d'établir à partir des données expérimentales publiées dans la littérature. On constate tout d'abord que les composants IMPATT sont les composants les plus performants d'un point de vue puissance de sortie $[9,10]$. Ainsi des différences d'un ordre de grandeur peuvent être mises en évidence entre les deux types de composant (typiquement à $94 \mathrm{GHz} 1 \mathrm{~W}$ pour les diodes IMPATT $100 \mathrm{~mW}$ pour les diodes à transfert électronique). L'utilisation de composants Gunn se justifie cependant si l'on considère l'aspect propriétés de bruit. Ensuite, il apparaît que seuls les composants IMPATT silicium permettent actuellement de couvrir le haut de la gamme des fréquences millimétriques. Si l'on considère, en effet, les performances des diodes IMPATT GaAs une chute relativement brutale des niveaux de puissance émise peut être mise en évidence au-dessus de $60 \mathrm{GHz}$ en accord avec l'analyse théorique précédente $[39,41]$. Dans ce contexte, il nous paraît vain d'espérer des puissances de sortie supérieures à $0,5 \mathrm{~W}$ à $94 \mathrm{GHz}$ à

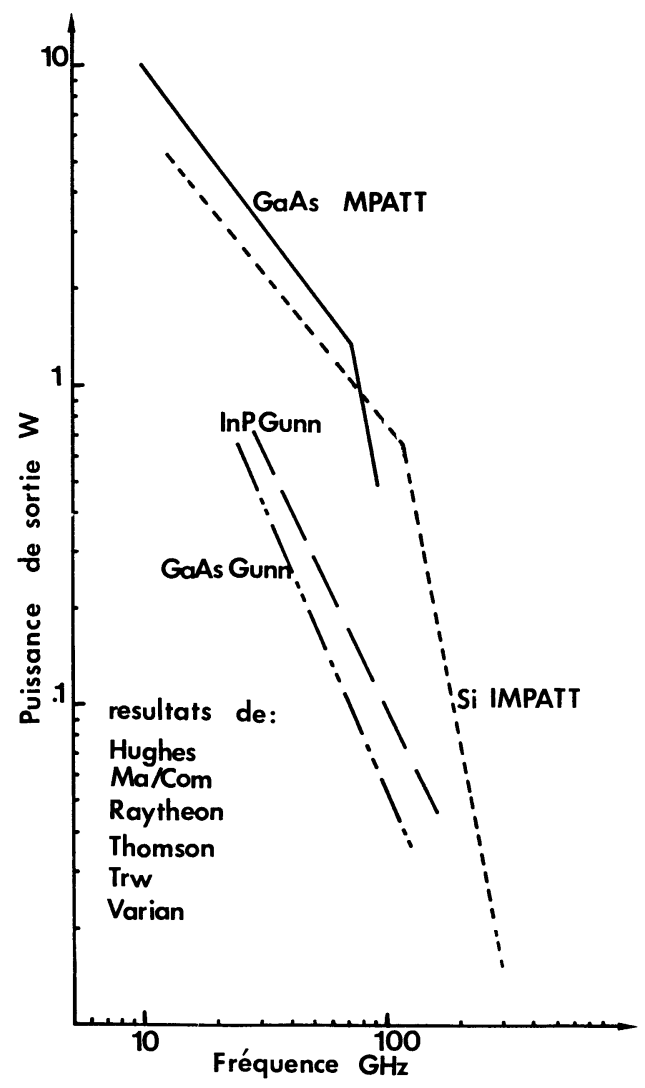

Fig. 18. - Evolution fréquentielle des puissances émises pour les composants dipôles.

[Frequency dependence of output power for two terminal devices.] 
l'aide de diodes IMPATT GaAs. Les informations relatives aux composants réels IMPATT InP [42] sont actuellement trop peu nombreuses pour figurer dans ce constat. Enfin, si l'on considère les composants à transfert électronique, la supériorité des diodes Gunn InP mise en évidence dans l'analyse théorique se retrouve au niveau des puissances émises par les composants réels [43, 44].

La figure 19(a) montre les variations de la puissance de sortie $P_{\mathrm{s}}$ fonction de la fréquence, établies à partir des performances expérimentales des composants MESFET de puissance [45]. Il est à noter que des valeurs de $P_{\mathrm{s}}$ de l'ordre du Watt sont obtenues à $20 \mathrm{GHz}$. On observe cependant une décroissance extrêmement abrupte de $P_{\mathrm{s}}$ qui varie approximativement en $1 / f^{3}$ au-dessus de cette fréquence. Il en résulte qu'aux fréquences millimétriques les valeurs nominales de la puissance ne sont plus que de quelques dizaines de milliwatts. Les gains en régime linéaire sont typiquement de $6 \mathrm{~dB}$ à $60 \mathrm{GHz}$. La figure 19(b) illustre l'évolution des niveaux de puissance de sortie en fonction de la fréquence pour des transistors TEGFET a multiples hétérojonctions issue des références [27-29]. Il est à noter que des performances comparables MESFET-TEGFET sont obtenues dans le bas de la bande millimétrique.

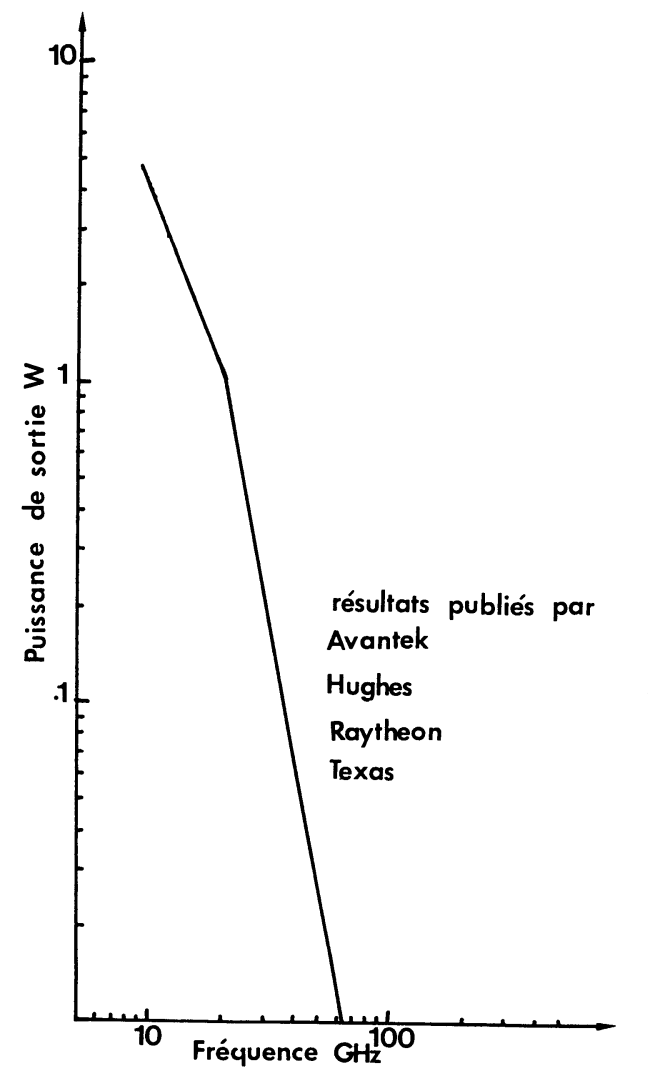

Fig. 19a. - Puissance délivrée par les composants tripôles MESFET de puissance.

[Frequency dependence of output power for MESFET.]

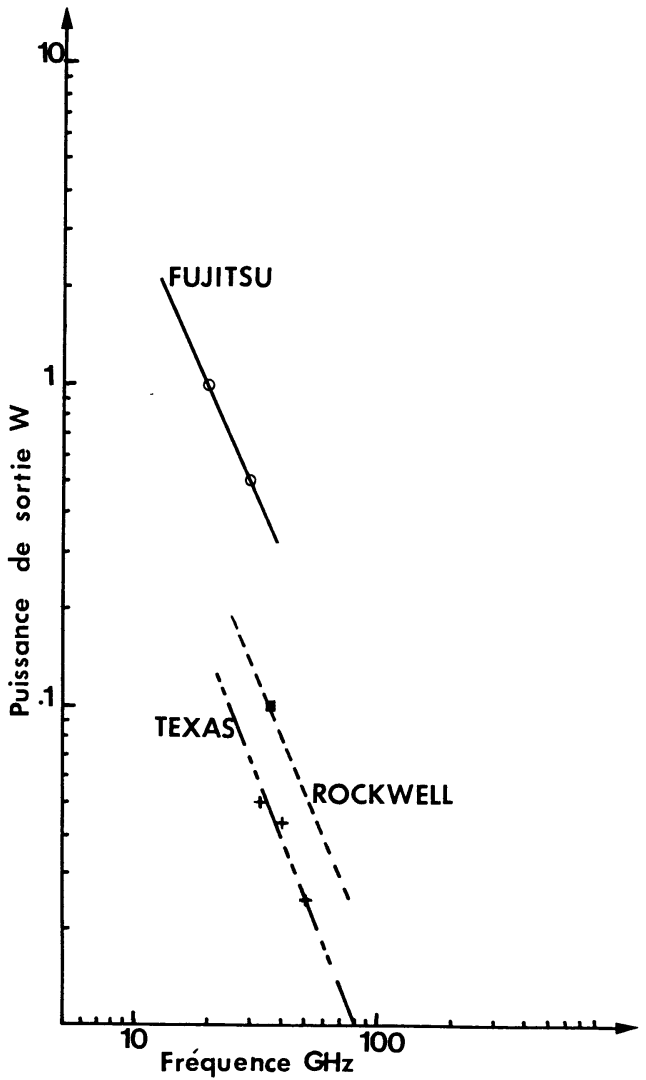

Fig. 19b. - Puissance de sortie des composants TEGFET à multiples hétérojonctions.

[Frequency dependence of output power for TEGFET.'

Cependant, il faut insister sur le fait que ces résultats ont été obtenus, pour les transistors fonctionnant à $60 \mathrm{GHz}$, avec des développements de grille faibles pour des composants de puissance. Si l'on considère les performances en termes de puissance normalisée l'on obtient des valeurs entre 0,4 et $0,6 \mathrm{~W} / \mathrm{mm}$ de grille. Sur cette base, on peut en conclure que le TEGFET de puissance devrait supplanter le MESFET aux ondes millimétriques.

\section{Structures et modes de fonctionnement nouveaux.}

4.1 IMPATT À HÉTÉROJONCTIONS ET MODE DE RELAXATION. - Les structures des composants à avalanche et temps de transit utilisant des hétérojonctions sont multiples. Nous les classerons en deux types. Nous considérerons d'une part les structures nouvelles qui visent à une amélioration des performances en jouant sur les propriétés de transit et d'autre part les structures dont la finalité est de pallier aux limitations inhérentes au phénomène de création paire électron-trou par ionisation par choc.

Pour le premier type de composant, il est principalement recherché un effet de modulation de la vitesse de transit des porteurs. En effet, on montre 
qu'une modulation de la vitesse de dérive des porteurs obtenue soit par variations extrêmement rapides du champ électrique appliqué [48] soit à l'aide de différents matériaux $[46,47]$ conduit à une augmentation du courant induit par le mécanisme de transit et donc des rendements de conversion. Les valeurs de rendements calculées pour des structures type $\mathrm{GaAs} / \mathrm{GaAlAs}$ sont encourageantes puisque des valeurs de l'ordre de $10 \%$ ont été déterminées à $80 \mathrm{GHz}$ pour un composant à simple zone de transit [46].

Pour le second type de composant, deux buts sont visés essentiellement. En premier lieu, il est recherché des structures permettant de confiner la zone de multiplication. Si l'on s'en réfère en effet à la figure 5 précédente, on constate un étalement important des densités de porteurs libres. Un tel élargissement est préjudiciable à l'obtention de rendements élevés. La figure 20 montre comment il est alors possible à partir d'hétéroépitaxies de restreindre la zone de multiplication à la zone centrale de GaAs [49]. On constate en effet que l'utilisation de couches de GaAlAs permet d'annuler pratiquement la probabilité d'ionisation (Fig. 20b). En second lieu, l'on peut espérer mettre à profit les discontinuités de bande interdite pour aider l'acquisition de l'énergie

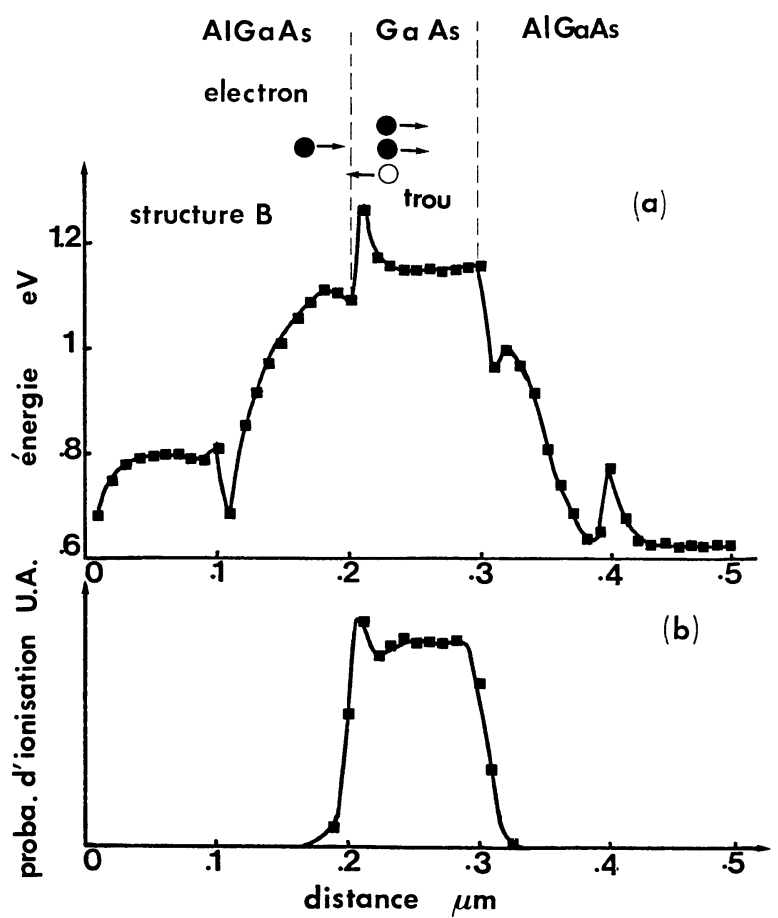

Fig. 20. - Hetero-IMPATT

(p-GaAs/p-Ga ${ }_{0,6} \mathrm{Al}_{0,4} \mathrm{As} / \mathrm{i}-\mathrm{GaAs} / \mathrm{n}-\mathrm{Ga}_{0,6} \mathrm{Al}_{0,4} \mathrm{As} / \mathrm{nGaAs}$ ) permettant le confinement de la zone d'avalanche.

\section{[Hetero-IMPATT}

(p-GaAs/p-Ga ${ }_{0.6} \mathrm{Al}_{0.4} / \mathrm{i}-\mathrm{GaAs} / \mathrm{n}-\mathrm{Ga}_{0.6} \mathrm{Al}_{0.4} \mathrm{As} / \mathrm{nGaAs}$ ) yielding avalanche zone confinement.] de seuil d'ionisation, comme cela a été démontré pour les photodiodes à avalanche [52]. Dans ces conditions, les tensions de fonctionnement nécessaires au claquage par avalanche sont plus faibles. Il est alors possible d'éviter la saturation des valeurs de coefficients d'ionisation mis en évidence pour les composants conventionnels. La structure de la figure 20 tout en n'étant pas optimisée présente des performances élevées (17\% de rendement d'émission dans la fenêtre $94 \mathrm{GHz}$ pour des structures à double zone de transit).

En outre un certain nombre d'études portent actuellement sur l'ionisation par choc dans les hétéroépitaxies multicouches $[50,51]$ qui peuvent être directement appliquées aux structures à zone d'avalanche à multi puits quantiques [53]. Dans un tout autre esprit, des études portent actuellement sur des structures à avalanche permettant de s'affranchir de la diminution importante des dimensions des zones actives à fréquences croissantes [54]. L'association de diodes à avalanche est en effet la solution $a$ priori la plus simple pour augmenter le niveau d'impédance et ajouter les puissances. En structure hybride cependant, cette association pose d'énormes problèmes de boîtier et de connectique. En conséquence, il s'avère beaucoup plus intéressant de mettre en série des composants élémentaires sur une même épitaxie. Pour que les diodes n'interagissent pas entre elles cependant il est nécessaire de les séparer par des couches métalliques. L'enjeu de réalisation de tels composants porte alors essentiellement sur la difficulté de reprise d'épitaxie métalsemiconducteur.

Enfin, quelques études ont été effectuées afin de déterminer dans quelle mesure des composants uniformement en avalanche (diodes $p-i-n)$ peuvent être utilisés aux fréquences millimétriques et submillimétriques [55, 56]. On peut montrer en effet que, pour ce cas où le phénomène d'avalanche est prépondérant, il est possible d'obtenir des résistances négatives pour des fréquences de l'ordre de $300 \mathrm{GHz}$ par effet de relaxation de l'énergie [55]. Ces effets sont illustrés sur la figure 21 qui met en évidence les relations de phase entre la tension aux bornes d'un composant $\mathrm{p}-\mathrm{i}-\mathrm{n}$ et la densité de porteurs libres par détermination des intercorrélations entre ces deux grandeurs.

A ces fréquences, en effet, l'énergie des porteurs qui est le paramètre fondamental pour la création de porteurs en excès par ionisation par choc ne peut suivre instantanément les variations temporelles du champ électrique. Il en résulte que le courant de porteurs créés peut être déphasé de plus de $90^{\circ}$ par rapport à la tension appliquée.

4.2 Diodes GunN À InJECTION CONTRÔléE. Nous avons vu au paragraphe 2 que pour les composants Gunn classiques $\mathrm{n}^{+} \mathrm{nn}^{+}$la longueur active de 


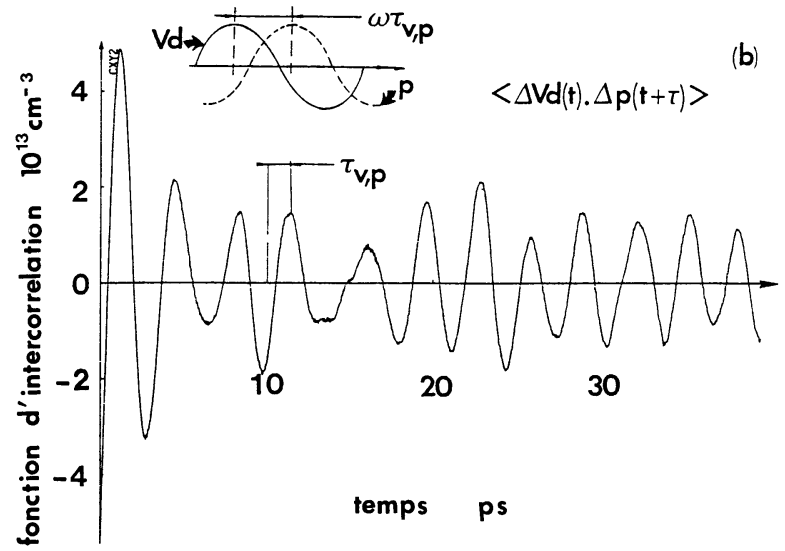

Fig. 21. - Diodes à avalanche utilisant les effets de relaxation de l'énergie : mise en évidence d'un déphasage tension-densité de porteurs libres supérieur à $\pi / 2$.

[Avalanche diode operation based on energy relaxation effect : illustration of a voltage-carrier density phase shift in excess of $\pi / 2$.]

l'échantillon est réduite d'une part par la présence de la zone morte et d'autre part par la nécessité de satisfaire à la condition de transit. Pour pallier ces difficultés, un certain nombre de structures nouvelles ont été proposées visant dans la plupart des cas à un contrôle de l'injection des porteurs dans la zone active. Nous nous limiterons ici aux solutions qui nous paraissent avoir le plus de chance d'aboutir. Nous allons considérer respectivement l'utilisation de contact limitant métal semiconducteur, d'hétérojonctions et de structures Gunn planar [57, 61].

La structure à contact limitant métal semiconducteur est très délicate à réaliser technologiquement compte tenu des problèmes de dérive en température. Le recuit doit être en effet optimisé afin d'obtenir un compromis entre une injection thermoionique et une injection de type contact injectant. Le rôle de ces contacts est tout d'abord de créer une injection de porteurs chauds dans la zone active de type $n$. On recherche ainsi une diminution de la zone morte et donc une augmentation des rendements d'émission. Par ailleurs, le contact métal semiconducteur est limitant et permet d'ajuster la valeur du courant injecté et donc la densité de charges libres au niveau de dopage de la zone active. On montre que dans ce cas des couches d'accumulation et de déplétion se forment au sein du composant [61]. Du fait du caractère dipolaire de la charge d'espace, les variations spatiales du champ électrique sont alors sensiblement uniformes [57]. Ces modes de fonctionnement à champ électrique quasi uniforme sont illustrés sur la figure 22 issue de la référence [61]. En pratique, il est alors possible de réaliser des composants dont les dimensions peuvent être 3 fois supérieures aux dimensions imposées par la condition de transit. A titre d'exemple, un niveau de puissance
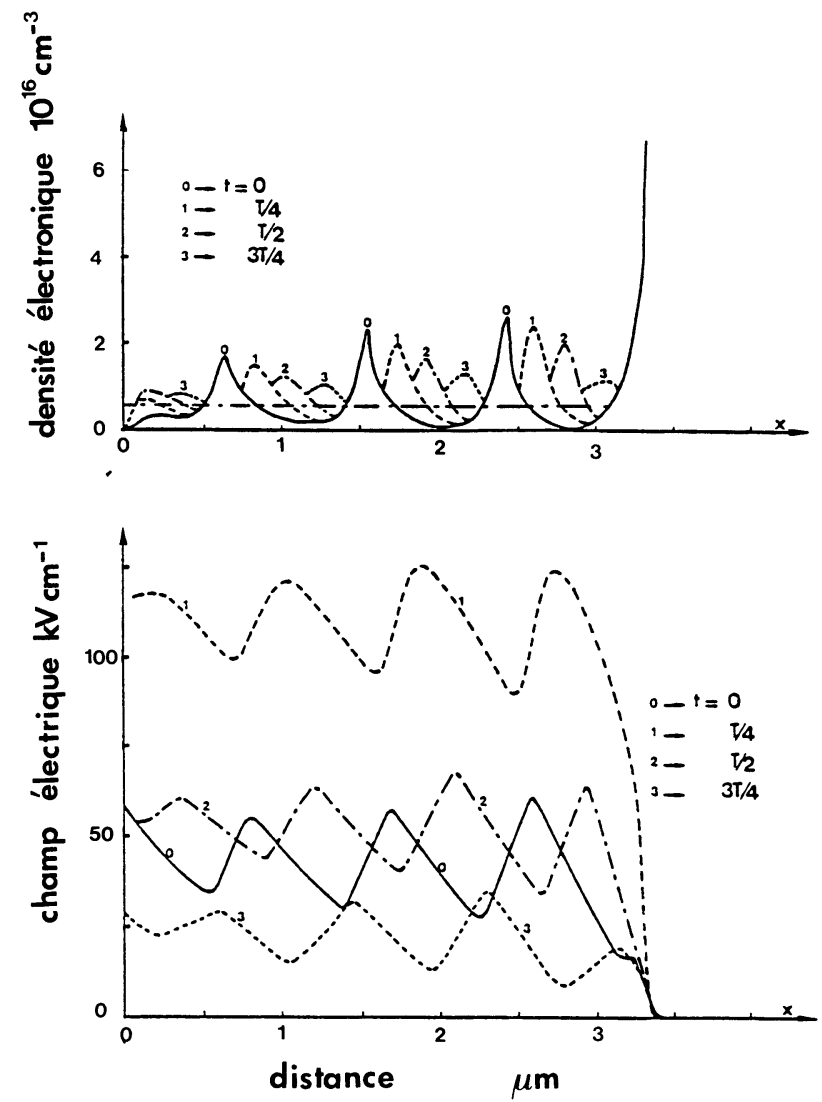

Fig. 22. - Illustration du fonctionnement des diodes Gunn à contact limitant.

[Illustration of injection-limited Gunn diodes.]

théorique de l'ordre de $200 \mathrm{~mW}$ avec un rendement de $10 \%$ pourrait être théoriquement obtenu à $94 \mathrm{GHz}$ pour un composant de $2,5 \mu \mathrm{m}$ de long.

Pour les structures à hétérojonction le contact est polarisé en inverse. Il en résulte la formation d'une zone de champ électrique élevée due à la présence de la zone désertée dans la zone active n. Dans cette région, les porteurs initialement froids acquièrent très rapidement l'énergie nécessaire au transfert dans les vallées satellites. La zone morte est dans ce cas réduite pratiquement à la longueur de la zone désertée dont les dimensions peuvent être de l'ordre de $0,1 \mu \mathrm{m}$. Ici encore, il est possible théoriquement d'ajuster le courant injecté en variant la composition des matériaux composant l'hétérojonction et ainsi fonctionner à champ électrique relativement uniforme [58]. Les puissances espérées sont de l'ordre de $150 \mathrm{~mW}$ pour un rendement de $7,5 \%$ dans la fenêtre $100 \mathrm{GHz}$ pour une structure Ga InAsP/InP.

Enfin la troisième solution consiste à contrôler l'injection des porteurs par une structure de type MESFET [59, 60]. Cette cathode MESFET conduit. à une structure de type Gunn planar. Par ajustement de la tension grille source on montre que dans ce cas il est possible de passer d'un mode de fonctionnement à couches d'accumulation à un mode de 
fonctionnement à couches dipolaires. Les niveaux de puissance espérés à l'aide de ces composants sont comparables à ceux obtenus avec des composants classiques $(100 \mathrm{~mW}, 4 \%$ à $100 \mathrm{GHz})$. Cette solution se justifie cependant par les possibilités d'intégration qui résultent de l'emploi d'une structure planar.

4.3 TRAnsistors FET À GRILle isoléE. - Au cours du paragraphe 2 nous avons vu que les performances en puissance des transistors FET sont limitées par les valeurs de tensions admissibles. En structure à canal creusé la tension $V_{\mathrm{ds}}$ maximum est limitée par le claquage par avalanche au niveau de la grille. De ce fait, il semble intéressant de concevoir des structures FET à grille isolée. Deux voies de recherche ayant trait respectivement aux transistors MISFET InP et MIS-like-FET GaAs/GaAlAs sont plus particulièrement développées actuellement. Nous allons les considérer respectivement.

Les avantages d'utilisation du matériau InP dans les réalisations de transistors FET sont triples [62]. Il présente en effet une vitesse électronique élevée, des valeurs de coefficients d'ionisation plus faibles que les matériaux $\mathrm{AsGa}$ et $\mathrm{Si}$, et une conductivité thermique importante. On peut s'attendre par conséquent à une augmentation de la fréquence de transit sous la grille et des tensions admissibles au niveau du composant. Par ailleurs, la réalisation de structure métal-oxyde-isolant outre les avantages mentionnés ci-dessus, permet d'annuler le courant de fuite de grille qui se révèle important pour les composants type MESFET InP. Pratiquement cependant, le dépôt d'oxydes sur InP fait intervenir une densité de pièges élevée. Il en résulte une variation importante de la valeur du courant $I_{\mathrm{ds}}$ à long terme (long drift term). La mobilité des porteurs mesurée expérimentalement est en outre assez faible comparativement aux structures plus conventionnelles. Les recherches sur ces composants s'attachent plus particulièrement à résoudre ces problèmes soit en améliorant les technologies propres aux oxydes, soit en essayant différents matériaux isolants [62, 63]. Des résultats expérimentaux ont été obtenus à ce jour principalement en bande $X$. A titre d'exemple une puissance de sortie normalisée de $4,2 \mathrm{~W} / \mathrm{mm}$ de grille a été mesurée à $9 \mathrm{GHz}$ [62]. Cette puissance est très largement supérieure à celle obtenue avec des FET AsGa montrant l'intérêt des structures MISFET InP dans l'amplification de puissance.

La structure des composants MIS-like-FET [67, 70] est schématisée sur la figure 23. Elle consiste principalement en une couche de GaAlAs non dopée, de $\mathrm{GaAs}$ très fortement dopée typiquement $10^{18} \mathrm{~cm}^{-3}$, de $\mathrm{GaAs}$ non dopé. En surface une couche de $\mathrm{GaAs}$ de faible épaisseur (50 $\mathrm{A})$ fortement dopée facilite la réalisation des contacts ohmiques. Par opposition aux composants FET de puissance dont la grille est réalisée sur couche semiconductrice

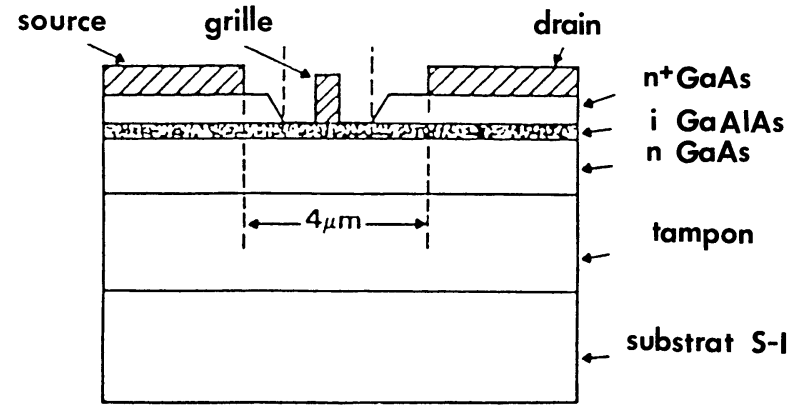

Fig. 23. - Structure « MIS-LIKE-FET ».

[« MIS-LIKE » FET Structure.]

fortement dopée, le contact schottky de grille est ici réalisé sur matériau peu dopé. On évite de ce fait les claquages dus aux valeurs de champ électrique élevées au niveau du contact schottky. Par ailleurs, la structure MIS-like-FET peut présenter des variations de la transconductance en fonction de $V_{\mathrm{gs}}$ moins marquées que celles mesurées typiquement pour les composants TEGFET. Un exemple de caractéristique $g_{\mathrm{m}}\left(V_{\mathrm{gs}}\right)$ est donné sur la figure 24 pour un composant TEGFET. On note une plage assez restreinte de valeurs de tensions $V_{\mathrm{gs}}$ pour lesquelles $g_{\mathrm{m}}$ est élevé. Cette évolution qui s'explique en particulier par le transport d'une partie des électrons dans la couche de GaAlAs entraîne que pour des excursions importantes de tension $V_{\mathrm{gs}}$ la

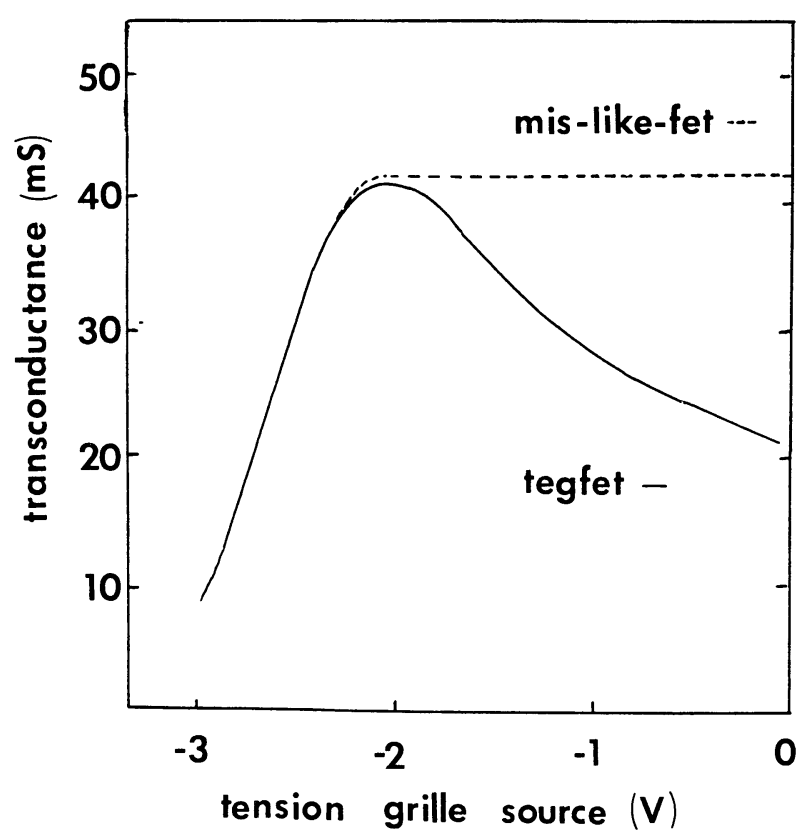

Fig. 24. - Variations typiques de la transconductance en fonction de $V_{\mathrm{gs}}$ pour un composant TEGFET et pour un « MIS-LIKE-FET ».

[Transconductance versus $V_{\mathrm{gs}}$ voltage for TEGFET and MIS-LIKE FET devices.] 
valeur moyenne de $g_{\mathrm{m}}$ est diminuée. La structure MIS-like-FET ne souffre pas de cette limitation et permet par conséquent d'améliorer les valeurs de courant admissibles. A titre d'exemple la valeur du courant de saturation pour un transistor MIS-likeFET de $0,5 \mu \mathrm{m}$ de longueur de grille est de $650 \mathrm{~mA} / \mathrm{mm}$ [68]. De plus, $1 \mathrm{~W} / \mathrm{mm}$ de densité de puissance a été obtenu à $18,5 \mathrm{GHz}$ [67]. Il n'est pas utopique de penser que de telles performances puissent être étendues à $30 \mathrm{GHz}$.

4.4 COMPOSANTS DISTRIBUÉs. - Nous nous limiterons à deux exemples de composants distribués susceptibles d'être utilisés dans les applications de puissance respectivement les composants IMPATT distribués (DIMPATT) et les amplificateurs FET distribués.

L'idée des composants DIMPATT est de considérer une structure continûment répartie constituant un milieu semiconducteur à conductivité négative et qui permettrait ainsi l'amplification d'une onde progressive [71]. Un exemple de structure basée sur ce principe est schématisé sur la figure 25 . Elle est constituée d'une jonction $\mathrm{p}^{+} \mathrm{n}$ que l'on polarise en régime d'avalanche. La propagation de l'onde qui peut être amplifiée ou émise suivant les conditions d'adaptation (structure auto-résonnante dans le cas d'un oscillateur) se fait suivant la direction $z$. Le principal intérêt de ces composants distribués provient de leur utilisation en mode à résonnance parallèle comparé au mode à résonnance série rencontré dans les composants mésa conventionnels. La faisabilité de ces dispositifs a été démontrée tant en centimétrique où des puissances de sortie de l'ordre de $2 \mathrm{~W}$ ont été obtenues avec $10 \mathrm{~dB}$ de gain [72] qu'en millimétrique avec une fréquence d'oscillations voisine de $90 \mathrm{GHz}$ [73].

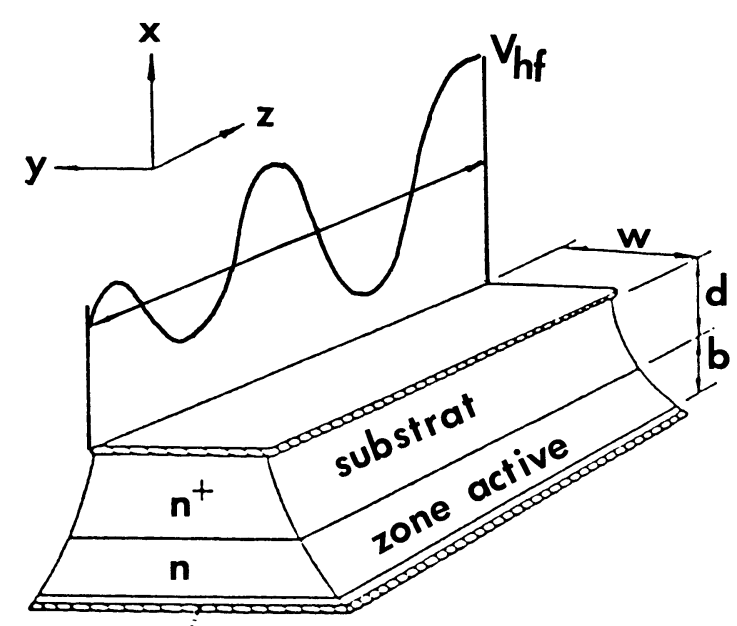

Fig. 25. - Structure D IMPATT.

[D IMPATT structure.]
Le principe de l'amplificateur FET distribué est d'intégrer les capacités d'entrée et 'de sortie de transistors FET dans une structure à deux lignes de transmission couplées par les transconductances des composants. L'égalité des vitesses de phases sur les lignes drain et grille est généralement obtenue par adjonction d'une capacité additionnelle. L'intérêt de cette association, outre la possibilité d'obtenir des gains théoriques élevés par l'association de plusieurs transistors (en première approximation $G$ est proportionnel à $n^{2} g_{\mathrm{m}}^{2}$ avec $n$ le nombre de transistors associés), est d'être adaptée à l'amplification large bande. A titre d'exemple, il a été démontré la possibilité de fonctionner pratiquement de $2 \mathrm{GHz}$ jusqu'à des fréquences proches de $30 \mathrm{GHz}$ avec un gain petit signal de l'ordre de $6 \mathrm{~dB}$ [74]. Il semble toutefois que l'extension de ces résultats aux fréquences millimétriques soit un enjeu relativement difficile à l'heure actuelle compte tenu des difficultés de réalisation de ces composants en technologie monolithique. Par ailleurs, signalons que des travaux visent à étudier et tirer parti des effets de propagation dans les structures FET continûment distribuées [75].

\section{Conclusion.}

En résumé de cette étude, les points suivants nous paraissent importants :

Pour les composants conventionnels ou au stade du développement

- Dans le bas de la gamme des ondes millimétriques les composants tripôles TEGFET et MESFET tendent de plus en plus à avoir des performances en puissance comparables aux composants dipôles (typiquement $1 \mathrm{~W}$ à $30 \mathrm{GHz}$ ). Les performances des transistors bipolaires à hétérojonction sont par contre encore dominées par des problèmes technologiques.

- Au-dessus de $100 \mathrm{GHz}$, les diodes IMPATT se révèlent les composants les plus puissants (typiquement $50 \mathrm{~mW}$ à $200 \mathrm{GHz}$ ). Le choix des composants Gunn peut se justifier cependant si l'on considère une application mettant à profit leur bruit plus faible (typiquement $100 \mathrm{~mW}$ à $100 \mathrm{GHz}$ ).

\section{Pour les nouveaux composants}

- Ils sont basés essentiellement sur les nouvelles possibilités offertes par les modulations de matériaux (hétéro-Gunn et hétéro-IMPATT) et/ou de dopage (MIS-like-FET et. MIS InP). Ils visent à l'émélioration des performances tant en fréquence qu'en puissance. Leur développement dépend essentiellement des progrès technologiques à venir.

- Les structures distribuées sont actuellement développées dans les applications où les problèmes d'adaptation sont cruciaux, amplification large bande 
notamment. Leur développement aux fréquences millimétriques reste problématique.

\section{Remerciements.}

Cet article a été rédigé après discussions avec
Mesdames G. Colomer, M. A. Di Forte Poisson et Messieurs F. Azan, P. Baudet, A. Cappy, G. Colomer, E. Constant, J. C. Dejaeger, J. Cremillet, P. Huguet, J. Lacombe, A. Marty, J. Pribetich, M. Rocchi, G. Salmer et A. Scavennec. Nous les en remercions.

\section{Bibliographie}

[1] SzE, S. M., Physics of Semiconductor Devices (John Wiley) 1981.

[2] Perdomo, J. and Lee, C. H., IEEE Trans. Electron Devices ED-305 (1983) 217.

[3] Kim, K. and Hess, K., J. Appl. Phys. 60 (1986) 2626.

[4] Lippens, D., Nieruchalski, J. L. et CONSTANT, E., IEEE Trans. Electron Devices ED-32 (1985) 2269.

[5] Elta, M. E. et Haddad, G. I., IEEE Trans. Electron Devices ED-25 (1978) 694.

[6] LipPens, D. et Constant, E., Electron. Lett. 17 (1981) 878.

[7] Blakey, P. A. et Froelich, R. K., Electron. Lett. 21 (1985) 28.

[8] Zhang, X., Freyer, J., WeimanN, G. and SCHLAPO, W., IEE Proceedings 131, (1984) 203.

[9] Midford, A. et BERNICK, R., IEEE Trans. Microwave Theory Tech. MTT-27 (1979) 483.

[10] Heitzmann, M. and Boudot, M., IEEE Trans. Electron Devices ED-30 (1983) 759.

[11] Rosen, A. et al., IEEE Trans. Microwave Theory Techn. MTT-30 (1982) 47.

[12] Lippens, D., Nieruchalski, J. L., Dalle, C. et ROLLAND, P. A., Int. J. Infrared and millimeter waves 7 (1986) 771.

[13] EdDison, I. G., Infrared and millimeter waves 11 (Academic Press) 1984.

[14] Rees, H. D., Supplément au J. Phys. C 7 (1981) 157.

[15] Rolland, P. A., Salmer, G., Constant, E. et FAUQuEMBERgue, R., IEEE Trans. Electron Devices ED-28 (1981) 341.

[16] Friscourt, M. R., Rolland, P. A., Cappy, A., Constant, E. et SAlmER, G., I.E.E.E. Trans Electron Devices ED-30 (1983) 223.

[17] Thim, H. W. and Poetzi, H. W., Inst. Phys. Conf. Ser. 321977

[18] Friscourt, M. R. et Rolland, P. A., IEEE Electron Devices Lett. EDL-4 (1983) 135.

[19] Ridley, B. K., J. Appl. Phys. 48 (1977) 754.

[20] Friscourt, M. R., Rolland, P. A. and FauquemBERGUE, R., IEEE Electron Devices Lett. EDL5 (1984) 434.

[21] Cappy, A., Carnez, B., Fauquembergues, R., SAlmer, G. et CONSTANT, E., IEEE Trans. Electron Devices ED-27 (1980) 2158.

[22] Wisseman, W. R., Proceedings of the internal workshop on Millimeter waves, avril 86 Rome.

[23] TsERng, H. Q., et KIM, B., Electron. Lett. 21 (1985) 178.

[24] Wroblewski, R., Salmer, G., Crosnier, Y. IEEE Trans. Electron Devices, ED-30 (1983) 154
[25] Crosnier, Y., Gerard, H., SAlMER, G., à paraître dans IEEE SSED.

[26] Cappy, A., VANOverschelde, A., ZimmermanN, J., Philippe, P., Versnayen, C. et Salmer, G., Revue Phys. Appl. 18 (1983) 719.

[27] SAUnier, P. et LEE, J. W., IEEE Electron Devices Lett. EDL-7 (1986) 503.

[28] Sheng, N. H., Lee, C. P., Chen, R. T., Miller, D. L., LEE, S. J., IEEE Electron Device Lett. EDL-6 (1985) 307.

[29] Hikosaka, K., Mirachi, Y., Mimura, T. et Ade, M., IEEE Electron Device Lett. EDL-6 (1985) 341.

[30] Fricke, K., et Hartnagel, H. L., IEEE Electron Device Lett. EDL-6 (1985) 151.

[31] Henderson, T., Aksun, M. I., Peng, C. K. et MORKOC, H., IEDM 86, p. 464-466.

[32] AsBECK, et al., IEEE Int. Electron Devices Meeting, Digest tech. Papers (1985) 19.9.

[33] Fischer, R. et MORKOC, H., IEEE Electron Device Lett. EDL-7 (1986) 359.

[34] MoLL, N., IEEE Cornell Conference Ithaca, Août 1985.

[35] Dubon Chevallier, C., Duchenois, A. M., Bresse, J. F. et ANKRI, L., Electron. Lett. 21 (1985) 614.

[36] Bailbe, J. P., Marty, A., Rey, G., Tasselli, J. et BouyahyAOUI, A., IEEE J. Solid-State Electron ED-32 (1985) 61.

[37] Chang, M. F., Asbeck, P. M., Wang, K. C., SulLiVAN, G. J., Miller, D. L., Electron. Lett. 22 (1986) 1173.

[38] Druelle, Y., Crosnier, Y., et Salmer, G., ESSDERC 83, Canterbury, Europhys. Conf. Abst. 7-ED30 (1983).

[39] Zhang, X., Freyer, J., Weiman, G., SchlapP, W., IEE Proceeding 131 (1984).

[40] Masse, D., Adlerstein, M. G., Holway, L. H., 15th European Microwave Conference, Paris (1985).

[41] Benko, E. et Trinh, T., Electron. Lett. (1984) 20.

[42] FAnK, F. B., Vichr, M., ZubeCK, I. V., Tringali, D. R. et Crowley, J. C., 8th Cornell Conference on microwave semi-conductor devices and circuit, Août 1981.

[43] Fank, F. B., Crowley, J. D., Boswell, M. C., Hang, C., Wolfert, Ph.., Tringali, D., CHING, L. Y., European microwave conference, Liège, Sept. 1984.

[44] Di ForTe, M. A. et al., Electron. Lett. 20 (1984) 25 26.

[45] Microwave Journal (1982) Octobre. 
[46] De Jaeger, J. C., Kozlowski, R. et Salmer, G., Electron. Lett. 20 (1984) 803.

[47] Adlerstein, M. G. et StATZ, H., IEEE Electron. Device ED-26 (1979) 817.

[48] Lippens, D., Constant, E., Friscourt, M. R., Rolland, P. A. et SAlmer, G., IEEE Electron. Device Lett. EDL-3 (1982) 213.

[49] Lippens, D., Nieruchalski, J. L. et Constant, E., Physica 134B (1985) 72.

[50] Ridley, B. K., IEE Proceedings 132 (1985) 177.

[51] Brennan, K., IEEE Trans. Electron Devices ED-33 (1986) 1502.

[52] Capasso, F., Tsang, W. T., Hutchinson, A. L. and Williams, G. F., Appl. Phys. Lett. 40 (1982) 38.

[53] Christou, A. and Warmazis, K., Appl. Phys. Lett. 48 (1986) 1446.

[54] Wang, K. L. et Li, G. P., Electron Device Lett. EDL-4 1983.

[55] Lippens, D. et Nieruchalski, J. L., Appl. Phys. Lett. 48 (1986) 1464.

[56] Aishima, A. et Funushima, Y., Appl. Phys. 56 (1984) 1086.

[57] Thim, H. W. and Poetzi, H. W., Inst. Phys. Conf. Ser. 32 (1977) 73.

[58] Friscourt, M. R., Rolland, P. A. and PerniseK, M., IEEE Electron Device Lett. EDL-6 (1985) 497.

[59] Rieder, G., ThIM, H., KuCH, R., LubKe, K., AEU 5/6 (1983) 217.

[60] Rolland, P. A., CAPPy, A., Friscourt, M. R., IEEE Electron Device Lett. EDL-6 (1985) 497.

[61] Friscourt, M. R. et Rolland, P. A., Physica 129B (1985) 527.
[62] Armand, M., Bui, D. V., Chevrier, J. et Linh, N. I., Rev. Tech. Thomson-CSF 16 (1984) 79.

[63] Dimitriou, P., Post, G., ScavenneC, A. et Duhamel, N., Physica 129B (1985) 399.

[64] Schachter, R. et al., Appl. Phys. Lett. 47 (1985) 272.

[65] Matsui, M., Hirayama, Y., Arai, F. et Sugano, T., IEEE Electron Device Lett. EDL-4 (1983) 308.

[66] Lile, D. L. et Taylor, M. J., J. Appl. Phys. 54 (1983) 260.

[67] Kim, B., Tserng, H. G. et SHIH, H. D., IEEE Electron Device Lett. EDL-7 (1986) 638.

[68] Hida, H., Окамото, A., Toyoshima, H. et OHATA, K., IEEE Electron Device Lett. EDL-7 (1986) 625.

[69] KIM, B., Tserns, H. Q. et SHIH, H. D., IEEE Electron Device Lett. EDL-5 (1534) 494.

[70] Maezawa, K., Mizutani, T., Arai, K. et YANAGAWA, F., IEEE Electron Device Lett. EDL-7 (1986) 454.

[71] MAINS, R. K. et HADDAD, G., IEEE Trans. Microwave Theory Tech. MTT-34 (1986) 965.

[72] Bayraktaroglu, B. et SHIH, H. D., IEEE Electron Device Lett. EDL-5 (1984) 466.

[73] BayrakTAROgLu, B. et SHIH, H. D., IEEE Electron Device Lett. EDL-4 (1983) 393.

[74] Niclas, K. B., Remba, R. D., Pereira, R. et CANTOS, B. D., IEEE Trans. MTT MTT-34 (1986) 427.

[75] Holden, A., Daniel, D., Davies, I., Oxley, C. et REES, M. D., IEEE Electron Devices ED-32 (1985) 61. 